\title{
MIXED METHODS FOR STATIONARY NAVIER-STOKES EQUATIONS BASED ON PSEUDOSTRESS-PRESSURE-VELOCITY FORMULATION
}

\author{
ZHIQIANG CAI AND SHUN ZHANG
}

\begin{abstract}
In this paper, we develop and analyze mixed finite element methods for the Stokes and Navier-Stokes equations. Our mixed method is based on the pseudostress-pressure-velocity formulation. The pseudostress is approximated by the Raviart-Thomas, Brezzi-Douglas-Marini, or Brezzi-DouglasFortin-Marini elements, the pressure and the velocity by piecewise discontinuous polynomials of appropriate degree. It is shown that these sets of finite elements are stable and yield optimal accuracy for the Stokes problem. For the pseudostress-pressure-velocity formulation of the stationary Navier-Stokes equations, the well-posedness and error estimation results are established. By eliminating the pseudostress variables in the resulting algebraic system, we obtain cell-centered finite volume schemes for the velocity and pressure variables that preserve local balance of momentum.
\end{abstract}

\section{INTRODUCTION}

Let $\Omega$ be a bounded, open, connected subset of $\mathcal{R}^{d}(d=2$ or 3$)$ with a Lipschitz continuous boundary $\partial \Omega$. Denote the outward unit vector normal to the boundary by $\mathbf{n}=\left(n_{1}, \ldots, n_{d}\right)$. Let $\mathbf{f}=\left(f_{1}, \ldots, f_{d}\right)$ be a given external body force defined in $\Omega$. Let $\mathbf{u}(\mathbf{x})=\left(u_{1}, \ldots, u_{d}\right)$ be the velocity vector field and $p$ the pressure. This paper studies mixed finite element methods for solving the stationary Navier-Stokes equations:

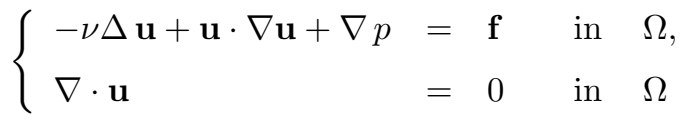

where $\nu>0$ is a given viscosity parameter and $\Delta, \nabla$, and $\nabla \cdot$ denote the Laplace, gradient, and divergence operators, respectively. The above system is supplemented with Dirichlet boundary conditions for the velocity

$$
\mathbf{u}=\mathbf{g} \quad \text { on } \partial \Omega
$$

where $\mathbf{g}=\left(g_{1}, \ldots, g_{d}\right)$ is a prescribed velocity on the boundary satisfying the compatibility condition

$$
\int_{\partial \Omega} \mathbf{n} \cdot \mathbf{g} d s=0
$$

Received by the editor January 15, 2010 and, in revised form, April 11, 2011.

2010 Mathematics Subject Classification. Primary 65M15, 65M60.

This work was supported in part by the National Science Foundation under grants DMS0511430 and DMS-0810855. 
Without the convection term, the system in (1.1) becomes the so-called Stokes equations:

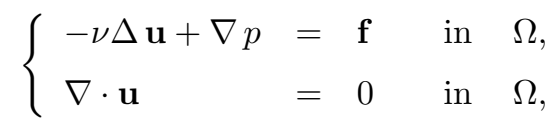

which is typically used for modeling creeping fluids flows and for studying numerical algorithms.

Numerical methods based on the velocity-pressure formulation in (1.1) have been extensively studied and well documented (see, e.g., books [10, 24, 25]). Recently, the practical need of the stress tensor associated with arising interest in non-Newtonian flows have motivated extensive studies of mixed finite element methods, stabilized mixed methods, and least-squares finite element methods based on the stressvelocity-pressure formulation or on its variants (see, e.g., 19, 20, 15, 15, 16, 23, 13 . and references therein). In [19, 20, Farhloul and Fortin introduced the gradient of the velocity, $\mathbf{t}=\nabla \mathbf{u}$, as a new independent variable and studied the corresponding hybridized mixed finite element method for the Stokes equation that uses the $R T_{0}$ element for the pseudostress $\boldsymbol{\sigma}=\nu \nabla \mathbf{u}-p \boldsymbol{\delta}$, the piecewise constants for the velocity and the pressure on elements, and the piecewise constants for the Lagrange multiplier on edges. In [19, this hybridized method was used for the Navier-Stokes equations by using the relation $(\mathbf{u} \cdot \nabla \mathbf{u}, \mathbf{v})=(\nabla \cdot(\mathbf{u} \otimes \mathbf{u}), \mathbf{v})$ for the nonlinear convection term, and the method was not analyzed mathematically. In [23, Gatica, González, and Meddahi studied a mixed finite element method by introducing both the gradient of the velocity and the pseudostress as new independent variables for the quasi-Newtonian Stokes flows. The pseudostress is approximated by the $R T_{0}$ element, the velocity and the gradient of the velocity are approximated by piecewise constants. In [5], Behr, Franca, and Tezduyar studied stabilized finite element methods based on the stress-pressure-velocity formulation for linearized incompressible Navier-Stokes equations. In [13, Cai, Lee, and Wang studied least-squares finite element methods based on the (pseudo)stress-velocity and the (pseudo)stress-velocity-pressure formulations.

Recently, in [15, 16] we analyzed a mixed finite element method based on the pseudostress-velocity formulation for solving the Stokes and Navier-Stokes equations. The pseudostress and the velocity are approximated by a stable pair of finite elements: Raviart-Thomas (RT) elements of index $k \geq 0$ and discontinuous piecewise polynomials of degree $k \geq 0$, respectively. As pointed out in [15], this method is accurate for approximations to the stress, the vorticity, and other physical quantities, preserves local balance of momentum, and has no essential boundary conditions. Moreover, the discrete algebraic system can be reduced to a smaller system having only the pseudostress unknowns through a penalty method by eliminating the velocity, and the resulting pseudostress system can be numerically solved by fast and efficient $H($ div) multigrid methods introduced in [3, 26, 30]. For the stationary Stokes equations, it is shown theoretically in [17] that the convergence rate of the $V(1,1)$-cycle multigrid is independent of the mesh size, the number of levels, and the penalty parameter. This is also confirmed numerically in [15, 17] with remarkably fast convergence rates around 0.21 for the RT element of index zero and 0.14 for the Brezzi-Douglas-Marini (BDM) element of index one.

The purpose of this paper is to study mixed finite element methods based on the pseudostress-pressure-velocity formulation for the incompressible Stokes and 
Navier-Stokes equations. Obviously, this formulation leads to a slightly larger system comparing to the pseudostress-velocity formulation due to the extra independent variable: the pressure. However, it is possible to reduce dramatically the size of the algebraic system by eliminating the pseudostress unknowns at the discrete level through the hybridization technique (see [22, 1]). This leads to discretization schemes of finite volume type that preserve local balance of momentum (see Section 6 ). For more details of this elimination procedure, see [12 for second-order elliptic equations.

In this paper, the pseudostress is approximated by the $H$ (div) conforming elements such as RT, BDM and Brezzi-Douglas-Fortin-Marini (BDFM) elements, and both the pressure and the velocity are approximated by piecewise discontinuous polynomials of appropriate degrees. For the Stokes equations, it is shown that these sets of finite elements are stable and yield optimal accuracy. To establish the stability and the optimality of the finite element approximations, in particular, the $L^{2}$ optimal accuracy of the $B D M$ elements, we make use of a fundamental inequality that bounds the $L^{2}$ norm of a tensor function by the $L^{2}$ norm of its deviatoric part and the $H^{-1}$ norm of its divergence. For the stationary Navier-Stokes equations, following our previous work in [16], we prove the well posedness of the pseudostress-pressure-velocity formulation via establishing its equivalence to the velocity-pressure formulation. Based on the theory of Brezzi, Rappaz, and Raviart [11, 24], we show that for sufficiently small mesh size, the discrete problem has a branch of nonsingular solutions in a neighborhood of the solution of the continuous problem. Moreover, we establish accuracy $O\left(h^{k+1-\frac{d}{6}}\right)$ in the $L^{3}(\Omega)$ norm. For a different analysis for nonlinear scalar elliptic equations in the mixed form, see [27] by Milner and Park.

The paper is organized as follows. The pseudostress-pressure-velocity formulation of Stokes equations is derived in Section 2. Section 3 describes and analyzes mixed finite element methods for the Stokes equations. Section 4 studies the stationary Navier-Stokes equations in the pseudostress-pressure-velocity formulation. In Section 5, we analyze the mixed finite element methods of the stationary NavierStokes equations. Finally, we describe cell-centered finite volume schemes obtained from eliminating the pseudostress variables in Section 6 .

1.1. Notation. Assume, throughout the paper, that the boundary of $\Omega$ is of class $\mathscr{C}^{2}$, a two-dimensional convex polygon, or convex polyhedra. We use the standard notations and definitions for the Sobolev spaces $W^{s, p}(\Omega)^{d}$ and $W^{s, p}(\partial \Omega)^{d}$ for $s \geq 0$ and $p \in[1, \infty]$. The standard associated inner products are denoted by $(\cdot, \cdot)_{s, p, \Omega}$ and $(\cdot, \cdot)_{s, p, \partial \Omega}$, and their respective norms are denoted by $\|\cdot\|_{s, p, \Omega}$ and $\|\cdot\|_{s, p, \partial \Omega}$. (We suppress the superscript $d$ because the dependence on dimension will be clear by context. We also omit the subscript $\Omega$ from the inner product and norm designation when there is no risk of confusion.) For $s=0, W^{s, p}(\Omega)^{d}$ coincides with $L^{p}(\Omega)^{d}$. Moreover, the space $W^{s, 2}(\Omega)^{d}$ will generally be written in the shorthand form $H^{s}(\Omega)^{d}$. Set

$$
W_{0}^{1,2}(\Omega):=\left\{q \in W^{1,2}(\Omega) \mid q=0 \text { on } \partial \Omega\right\}
$$

and

$$
L_{0}^{2}(\Omega)=\left\{q \in L^{2}(\Omega) \mid \int_{\Omega} q d x=0\right\}
$$


Denote by $\mathscr{D}(\Omega)$ the linear space of infinitely differentiable functions with compact support on $\Omega$. Let

$$
H(\operatorname{div} ; \Omega)=\left\{\mathbf{v} \in L^{2}(\Omega)^{d} \mid \nabla \cdot \mathbf{v} \in L^{2}(\Omega)\right\},
$$

which is a Hilbert space equipped with the norm

$$
\|\mathbf{v}\|_{H(\operatorname{div})}=\left(\|\mathbf{v}\|_{0,2}^{2}+\|\nabla \cdot \mathbf{v}\|_{0,2}^{2}\right)^{\frac{1}{2}} .
$$

Next, we will introduce a space which is an analog of $H(\operatorname{div} ; \Omega)$ and is used for the solution space of the pseudostress. For any $s>2$, let

$$
W^{0, s}(\operatorname{div} ; \Omega)=\left\{\mathbf{v} \in L^{s}(\Omega)^{d} \mid \nabla \cdot \mathbf{v} \in L^{2}(\Omega)\right\} \subset H(\operatorname{div} ; \Omega)
$$

which is a Banach space equipped with the norm

$$
\|\mathbf{v}\|_{W^{0, s}(\operatorname{div})}=\left(\|\mathbf{v}\|_{0, s}^{2}+\|\nabla \cdot \mathbf{v}\|_{0,2}^{2}\right)^{\frac{1}{2}} .
$$

Finally, denote the collections of tensor functions in $H(\operatorname{div} ; \Omega)^{d}$ and $\widehat{W}^{0, s}(\operatorname{div} ; \Omega)^{d}$ whose trace has zero mean value by

$$
\widehat{H}(\operatorname{div} ; \Omega)^{d}=\left\{\boldsymbol{\tau} \in H(\operatorname{div} ; \Omega)^{d} \mid \int_{\Omega} \operatorname{tr} \boldsymbol{\tau} d x=0\right\}
$$

and

respectively.

$$
\widehat{W}^{0, s}(\operatorname{div} ; \Omega)^{d}=\left\{\boldsymbol{\tau} \in W^{0, s}(\operatorname{div} ; \Omega)^{d} \mid \int_{\Omega} \operatorname{tr} \boldsymbol{\tau} d x=0\right\},
$$

\section{Pseudostress-Pressure-Velocity formulation for Stokes equations}

Introducing a new independent tensor variable, pseudostress:

$$
\boldsymbol{\sigma}=\nu \nabla \mathbf{u}-p \boldsymbol{\delta},
$$

where $\boldsymbol{\delta}$ denotes the identity tensor, then the first equation in (1.4) becomes

$$
-\nabla \cdot \boldsymbol{\sigma}=\mathbf{f} .
$$

Taking trace of (2.1) and using the second equation of (1.4) gives

$$
\operatorname{tr} \boldsymbol{\sigma}=-d p .
$$

Hence, we have the following pseudostress-pressure-velocity formulation of the Stokes equation:

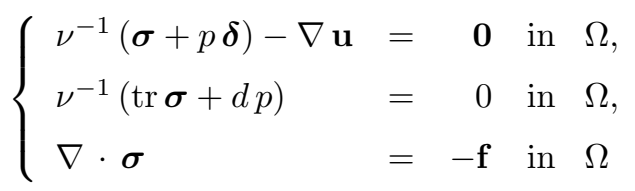

with boundary conditions (1.2) and compatibility condition (1.3). Note that the incompressibility condition is implicitly contained in the first and second equations of (2.4). Note also that elimination of the pressure and the second equation in (2.4) leads to the pseudostress-velocity formulation whose numerical methods were previously studied in [15]. 
Multiplying the first equation in (2.4) by a tensor function $\boldsymbol{\tau} \in \widehat{H}(\operatorname{div} ; \Omega)^{d}$, integrating over the domain $\Omega$, and using integration by parts and boundary conditions (1.2) gives

$$
\frac{1}{\nu}(\boldsymbol{\sigma}+p \boldsymbol{\delta}, \boldsymbol{\tau})+(\mathbf{u}, \nabla \cdot \boldsymbol{\tau})=\int_{\partial \Omega} \mathbf{g} \cdot(\mathbf{n} \cdot \boldsymbol{\tau}) d s \equiv g(\boldsymbol{\tau}) .
$$

Multiplying the second and third equations by a scalar function $q \in L^{2}(\Omega)$ and a vector function $\mathbf{v} \in L^{2}(\Omega)^{d}$, respectively, and integrating them over the domain $\Omega$ yields

$$
\frac{1}{\nu}(\boldsymbol{\sigma}+p \boldsymbol{\delta}, q \boldsymbol{\delta})=\frac{1}{\nu}(\operatorname{tr} \boldsymbol{\sigma}+d p, q)=0 \quad \text { and } \quad(\nabla \cdot \boldsymbol{\sigma}, \mathbf{v})=-(\mathbf{f}, \mathbf{v}) \equiv f(\mathbf{v}) .
$$

Let

$$
X^{d}:=\widehat{H}(\operatorname{div} ; \Omega)^{d} \times L^{2}(\Omega)
$$

equipped with the standard product norm

$$
\|(\boldsymbol{\tau}, q)\|=\|\boldsymbol{\tau}\|_{H(\text { div })}+\|q\|_{0,2}, \quad \forall(\boldsymbol{\tau}, q) \in X^{d} .
$$

Define the bilinear forms $a(\cdot, \cdot): X^{d} \times X^{d} \rightarrow \mathcal{R}$ and $b(\cdot, \cdot): X^{d} \times L^{2}(\Omega)^{d} \rightarrow \mathcal{R}$ by

$$
a((\boldsymbol{\sigma}, p),(\boldsymbol{\tau}, q))=\frac{1}{\nu}(\boldsymbol{\sigma}+p \boldsymbol{\delta}, \boldsymbol{\tau}+q \boldsymbol{\delta}) \quad \text { and } \quad b((\boldsymbol{\sigma}, p), \mathbf{v})=(\nabla \cdot \boldsymbol{\sigma}, \mathbf{v})
$$

respectively. Then the variational problem of the pseudostress-pressure-velocity formulation is to find $((\boldsymbol{\sigma}, p), \mathbf{u}) \in X^{d} \times L^{2}(\Omega)^{d}$ such that

$$
\left\{\begin{array}{lll}
a((\boldsymbol{\sigma}, p),(\boldsymbol{\tau}, q))+b((\boldsymbol{\tau}, q), \mathbf{u}) & =g(\boldsymbol{\tau}) & \forall(\boldsymbol{\tau}, q) \in X^{d}, \\
b((\boldsymbol{\sigma}, p), \mathbf{v}) & =f(\mathbf{v}) & \forall \mathbf{v} \in L^{2}(\Omega)^{d} .
\end{array}\right.
$$

To study the well-posedness of (2.6) and estimate error bounds of finite element approximations, we will need the following inequality. (In this paper, we use $C$ with or without subscripts to denote a generic positive constant, possibly different at different occurrences; that is, independent of the mesh size $h$ but may depend on the domain $\Omega$.)

Lemma 2.1. For any $\boldsymbol{\tau} \in \widehat{H}(\operatorname{div} ; \Omega)^{d}$, there exists a positive constant $C$ such that

$$
\|\boldsymbol{\tau}\|_{0,2} \leq C\left(\|\mathcal{A} \boldsymbol{\tau}\|_{0,2}+\|\nabla \cdot \boldsymbol{\tau}\|_{-1,2}\right),
$$

where $\mathcal{A} \boldsymbol{\tau}=\boldsymbol{\tau}-\frac{1}{d}(\operatorname{tr} \boldsymbol{\tau}) \boldsymbol{\delta}$ is the deviatoric part of the tensor $\boldsymbol{\tau}$.

Proof. It was shown (see Lemma 3.2 in [14] with $\mu=1 / 2$ ) that there exists a constant $C>0$ independent of $\lambda$ such that

$$
\|\boldsymbol{\tau}\|_{0,2} \leq C\left(\left(\mathcal{A}_{\lambda} \boldsymbol{\tau}, \boldsymbol{\tau}\right)^{1 / 2}+\|\nabla \cdot \boldsymbol{\tau}\|_{-1,2}\right),
$$

where $\mathcal{A}_{\lambda} \boldsymbol{\tau}=\boldsymbol{\tau}-\frac{\lambda}{d \lambda+1}(\operatorname{tr} \boldsymbol{\tau}) \boldsymbol{\delta}=\mathcal{A} \boldsymbol{\tau}+\frac{1}{d(d \lambda+1)}(\operatorname{tr} \boldsymbol{\tau}) \boldsymbol{\delta}$. Since $\|\mathcal{A} \boldsymbol{\tau}\|_{0,2}^{2}=$ $(\mathcal{A} \tau, \tau),(2.7)$ is then a limit of (2.8) as $\lambda \rightarrow \infty$.

For the convenience of readers, we sketch the proof of (2.8) for three dimensions $(d=3)$. A simple calculation gives

$$
\|\boldsymbol{\tau}\|_{0,2}^{2}=\left(\mathcal{A}_{\lambda} \boldsymbol{\tau}, \boldsymbol{\tau}\right)+\frac{\lambda}{3 \lambda+1}\|\operatorname{tr} \boldsymbol{\tau}\|_{0,2}^{2} .
$$


Then it suffices to show that there exists a constant $C>0$ independent of $\lambda$ such that

$$
\|\operatorname{tr} \boldsymbol{\tau}\|_{0,2} \leq C\left(\left(\mathcal{A}_{\lambda} \boldsymbol{\tau}, \boldsymbol{\tau}\right)^{1 / 2}+\|\nabla \cdot \boldsymbol{\tau}\|_{-1,2}\right) .
$$

This is established in Lemma 5.4 of [14 through the Helmholtz decomposition:

$$
\boldsymbol{\tau}=\mathcal{A}_{\lambda}^{-1} \nabla \mathbf{q}+\nabla \times \mathbf{\Phi}=\nabla \mathbf{q}+\lambda(\nabla \cdot \mathbf{q}) \boldsymbol{\delta}+\nabla \times \mathbf{\Phi},
$$

where $\mathbf{q} \in W_{0}^{1,2}(\Omega)^{3}$ and $\boldsymbol{\Phi} \in H(\operatorname{curl} ; \Omega)^{3}$ satisfy the following inequalities (Lemma 5.2 and (5.12) and (5.14) in [14]):

$$
\lambda\|\nabla \cdot \mathbf{q}\|_{0,2} \leq C\|\nabla \cdot \boldsymbol{\tau}\|_{-1,2} \quad \text { and } \quad\|\operatorname{tr} \nabla \times \boldsymbol{\Phi}\|_{0,2} \leq C\left\|\mathcal{A}_{\lambda}^{-1 / 2} \boldsymbol{\tau}\right\|_{0,2} .
$$

Now, (2.9) follows from the identity $\operatorname{tr} \boldsymbol{\tau}=(1+3 \lambda) \nabla \cdot \mathbf{q}+\operatorname{tr} \nabla \times \mathbf{\Phi}$ and the triangle inequality.

Remark 2.2. A slightly weaker result, $\|\boldsymbol{\tau}\|_{0,2} \leq C\left(\|\mathcal{A} \boldsymbol{\tau}\|_{0,2}+\|\nabla \cdot \boldsymbol{\tau}\|_{0,2}\right)$ in two dimensions was proved in [2] and 10] (Prop. 3.1 in Chap. IV). Note that the negative norm in (2.7) is essential for showing the optimal convergence rate of BDM elements.

To prove the uniqueness and existence of our variational problem, we employ the abstract theory on saddle-point problems in [6, 10. To this end, denote by $B: X^{d} \rightarrow L^{2}(\Omega)^{d}$ the linear operator associated with the bilinear form $b(\cdot, \cdot)$; i.e., for any $\boldsymbol{\tau} \in X^{d}, B \boldsymbol{\tau} \in L^{2}(\Omega)^{d}$ satisfies

$$
(B \boldsymbol{\tau}, \mathbf{v})=b(\boldsymbol{\tau}, \mathbf{v}) \quad \forall \mathbf{v} \in L^{2}(\Omega)^{d} .
$$

Denote the divergence-free subspace of $\widehat{H}(\operatorname{div} ; \Omega)^{d}$ by

$$
\hat{H}^{0}(\operatorname{div} ; \Omega)^{d}=\left\{\boldsymbol{\tau} \in \widehat{H}(\operatorname{div} ; \Omega)^{d} \mid \nabla \cdot \boldsymbol{\tau}=\mathbf{0}\right\},
$$

then it is easy to see that

$$
\operatorname{Ker} B=X^{0, d}=\hat{H}^{0}(\operatorname{div} ; \Omega)^{d} \times L^{2}(\Omega) .
$$

The next lemma establishes the coercivity of the bilinear form $a(\cdot, \cdot)$ in $X^{0, d} \times X^{0, d}$.

Lemma 2.3. There exists a positive constant $\alpha$ such that

$$
\alpha\|(\boldsymbol{\tau}, q)\|^{2} \leq a((\boldsymbol{\tau}, q),(\boldsymbol{\tau}, q))
$$

for any $(\boldsymbol{\tau}, q)$ in $X^{0, d}$.

Proof. For any $(\boldsymbol{\tau}, q) \in X^{d}=\widehat{H}(\operatorname{div} ; \Omega)^{d} \times L^{2}(\Omega)$, it follows from the definition of $\mathcal{A} \tau$ and the triangle inequality that

$$
\|\mathcal{A} \boldsymbol{\tau}\|_{0,2} \leq\|\boldsymbol{\tau}+q \boldsymbol{\delta}\|_{0,2}+\left\|\frac{1}{d}(\operatorname{tr} \boldsymbol{\tau}+d q) \boldsymbol{\delta}\right\|_{0,2} \leq C\|\boldsymbol{\tau}+q \boldsymbol{\delta}\|_{0,2}
$$

and that

$$
\|q\|_{0,2} \leq\|\boldsymbol{\tau}+q \boldsymbol{\delta}\|_{0,2}+\|\boldsymbol{\tau}\|_{0,2}
$$

together with Lemma 2.1 implies

$$
\|\boldsymbol{\tau}\|_{0,2}+\|q\|_{0,2} \leq C\left(\|\boldsymbol{\tau}+q \boldsymbol{\delta}\|_{0,2}+\|\nabla \cdot \boldsymbol{\tau}\|_{-1,2}\right) .
$$

Hence,

$$
\|\boldsymbol{\tau}\|_{0,2}^{2}+\|q\|_{0,2}^{2} \leq C\left(a((\boldsymbol{\tau}, q),(\boldsymbol{\tau}, q))+\|\nabla \cdot \boldsymbol{\tau}\|_{-1,2}^{2}\right) .
$$


Now, for any $(\boldsymbol{\tau}, q) \in X^{0, d}=\hat{H}^{0}(\operatorname{div} ; \Omega)^{d} \times L^{2}(\Omega)$, the lemma is then a straightforward consequence of the face that $\nabla \cdot \boldsymbol{\tau}=\mathbf{0}$. This completes the proof of the lemma.

Theorem 2.4. The variational problem in (2.6) has a unique solution. Moreover, the solution satisfies the following a priori estimate:

$$
\|(\boldsymbol{\sigma}, p)\| \mid\|\mathbf{u}\|_{0,2} \leq C\left(\|\mathbf{f}\|_{0,2}+\|\mathbf{g}\|_{1 / 2,2, \partial \Omega}\right) .
$$

Proof. It is easy to see that the linear forms $f(\mathbf{v})$ and $g(\boldsymbol{\tau})$ are continuous in $L^{2}(\Omega)^{d}$ and $X^{d}$ :

$$
|f(\mathbf{v})| \leq\|\mathbf{f}\|_{0,2}\|\mathbf{v}\|_{0,2} \quad \text { and } \quad|g(\boldsymbol{\tau})| \leq\|\mathbf{g}\|_{1 / 2,2, \partial \Omega}\|\boldsymbol{\tau}\|_{H(\text { div })} \leq\|\mathbf{g}\|_{1 / 2,2, \partial \Omega}\|(\boldsymbol{\tau}, q)\| \| .
$$

For any $\mathbf{v} \in L^{2}(\Omega)^{d}$, it was proved in [15, that there exists a constant $\beta>0$, such that

$$
\sup _{\boldsymbol{\tau} \in \widehat{H}(\operatorname{div} ; \Omega)^{d}} \frac{(\nabla \cdot \boldsymbol{\tau}, \mathbf{v})}{\|\boldsymbol{\tau}\|_{H(\operatorname{div})}} \geq \beta\|\mathbf{v}\|_{0,2} .
$$

Together with the fact that

$$
\sup _{(\boldsymbol{\tau}, q) \in X^{d}} \frac{(\nabla \cdot \boldsymbol{\tau}, \mathbf{v})}{\|\mid(\boldsymbol{\tau}, q)\|} \geq \sup _{(\boldsymbol{\tau}, 0) \in X^{d}} \frac{(\nabla \cdot \boldsymbol{\tau}, \mathbf{v})}{\|\boldsymbol{\tau}\|_{H(\operatorname{div})}} \geq \sup _{\boldsymbol{\tau} \in \widehat{H}(\operatorname{div} ; \Omega)^{d}} \frac{(\nabla \cdot \boldsymbol{\tau}, \mathbf{v})}{\|\boldsymbol{\tau}\|_{H(\operatorname{div})}}
$$

yields

$$
\sup _{(\boldsymbol{\tau}, p) \in X^{d}} \frac{b((\boldsymbol{\tau}, q), \mathbf{v})}{\|\mid(\boldsymbol{\tau}, q)\|} \geq \beta\|\mathbf{v}\|_{0,2}
$$

for any $\mathbf{v} \in L^{2}(\Omega)^{d}$. Now, by the abstract theory in $[6$, the existence and uniqueness of the variational problem (2.6) and the a priori estimates (2.12) are immediate consequences of the continuity of the linear forms $f$ and $g$, the coercivity (2.10), and the inf-sup condition (2.13).

As pointed out in [15], the pseudostress contains more information than the stress

$$
\tilde{\boldsymbol{\sigma}}=-p \boldsymbol{\delta}+\nu\left(\nabla \mathbf{u}+(\nabla \mathbf{u})^{t}\right)=\boldsymbol{\sigma}+\nu(\nabla \mathbf{u})^{t} .
$$

Physical quantities such as the velocity gradient, stress, and vorticity can be algebraically expressed in terms of the pseudostress and pressure:

$$
\nabla \mathbf{u}=\frac{1}{\nu}(\boldsymbol{\sigma}+p \boldsymbol{\delta}), \quad \tilde{\boldsymbol{\sigma}}=\left(\boldsymbol{\sigma}+\boldsymbol{\sigma}^{t}\right)+p \boldsymbol{\delta}, \quad \text { and } \quad \boldsymbol{\omega}=\frac{1}{2 \nu}\left(\boldsymbol{\sigma}+\boldsymbol{\sigma}^{t}\right),
$$

respectively. Therefore, these physical quantities (if needed) can be computed in a post-processing procedure without degrading the accuracy of the approximation.

\section{Finite element approximation: Stokes equations}

Assume the domain $\Omega$ is polygonal (polyhedronal) in two (three) dimensions and that $\mathcal{T}_{h}$ is a quasi-regular triangulation of $\Omega$ into triangles or rectangles (tetrahedrons or cubes) in two (three) dimensions with size $O(h)$. Rectangular (cubic) elements are constructed on a reference element $\hat{K}=(-1,1)^{d}$ and then extended to general quadrilaterals through the change of variables (see [10]).

We approximate each row of the pseudostress by conforming elements of $\widehat{H}(\operatorname{div} ; \Omega)$, such as Raviart-Thomas (RT), Brezzi-Douglas-Marini (BDM), and Brezzi-Douglas-Fortin-Marini (BDFM) elements, and approximate the pressure and 
velocity by discontinuous polynomials of appropriate degrees. To this end, we describe these finite element spaces and their approximation properties by following [10]. Denote spaces of polynomials on an element $K \subset \mathcal{R}^{d}$ by:

$$
\begin{aligned}
& P_{k}(K) \text { is the space of polynomials of degree } \leq k \text {, } \\
& P_{k_{1}, k_{2}}(K)=\left\{p\left(x_{1}, x_{2}\right) \mid p\left(x_{1}, x_{2}\right)=\sum_{i \leq k_{1}, j \leq k_{2}} a_{i j} x_{1}^{i} x_{2}^{j}\right\}, \quad d=2 ; \\
& P_{k_{1}, k_{2}, k_{3}}(K)=\left\{p\left(x_{1}, x_{2}, x_{3}\right) \mid p\left(x_{1}, x_{2}, x_{3}\right)\right. \\
& \left.=\sum_{i \leq k_{1}, j \leq k_{2}, k \leq k_{3}} a_{i j k} x_{1}^{i} x_{2}^{j} x_{3}^{k}\right\}, \quad d=3 ; \\
& Q_{k}(K)= \begin{cases}P_{k, k}(K) & \text { for } d=2, \\
P_{k, k, k}(K) & \text { for } d=3 .\end{cases}
\end{aligned}
$$

Denote polynomial spaces on the boundary of an element $K$ by

$$
R_{k}(\partial K)=\left\{\phi \in L^{2}(\partial K)|\phi|_{e_{i}} \in P_{k}\left(e_{i}\right) \quad \forall e_{i} \in \partial K\right\},
$$

where $e_{i}(i=1, \ldots, 3$ for triangular elements, $i=1, \ldots, 4$ for tetrahedra or rectangular elements, or $i=1, \ldots, 6$ for cubic elements) are the edges or faces of $K$.

Let $\mathbf{x}=\left(x_{1}, \ldots, x_{d}\right)$. Define the local RT space of index $k \geq 0$ on an element $K$ $(29,28])$ by

$$
R T_{k}(K)= \begin{cases}P_{k}(K)^{d}+\mathbf{x} P_{k}(K), & K=\text { triangle/tetrahedron } \\ Q_{k}(K)^{d}+\mathbf{x} Q_{k}(K), & K=\text { rectangle/cube }\end{cases}
$$

Define the local BDM space of index $k \geq 1$ on a triangle or a tetrahedra $K([9,7])$ by

$$
B D M_{k}(K)=P_{k}(K)^{d},
$$

The local $B D M_{k}(K)$ of index $k \geq 1$ is defined on a rectangle (9]) by

$$
\begin{array}{r}
B D M_{k}(K)=\left\{\mathbf{q} \mid \mathbf{q}=\mathbf{p}_{k}+r \nabla^{\perp}\left(x_{1}^{k+1} x_{2}\right)+s \nabla^{\perp}\left(x_{1} x_{2}^{k+1}\right),\right. \\
\left.\forall \mathbf{p}_{k} \in P_{k}^{2}, \quad \forall r, s \in \mathcal{R}\right\}
\end{array}
$$

and on a cube $([7])$ by

$$
\begin{array}{r}
B D M_{k}(K)=\left\{\mathbf{q} \mid \mathbf{q}=\mathbf{p}_{k}+\sum_{i=0}^{k}\left[r_{i} \nabla \times\left\{0,0, x_{1} x_{2}^{i+1} x_{3}^{k-i}\right\}+s_{i} \nabla \times\left(x_{2} x_{3}^{k+1} x_{1}^{k-i}, 0,0\right)\right.\right. \\
\left.\left.+t_{i} \nabla \times\left(0, x_{3} x_{1}^{k+1} x_{2}^{k-i}, 0\right)\right], \quad \forall \mathbf{p}_{k} \in P_{k}^{3}, \quad \forall r_{i}, s_{i}, t_{i} \in \mathcal{R}\right\}
\end{array}
$$


Define the local BDFM space of index $k \geq 1$ on an element $K([]$ ) by

$$
B D F M_{k}(K)=\left\{\mathbf{v} \in B D F M_{k}(K)|\mathbf{v} \cdot \mathbf{n}|_{\partial K} \in R_{k-1}(\partial K)\right\},
$$

where $\mathbf{n}$ is the unit outward normal to $\partial K$. From now on, whenever it may be convenient, we will use $M_{k}(K)$ to denote $R T_{k}$ or $B D F M_{k+1}(K)$ for $k \geq 0$ or $B D M_{k}(K)$ for $k \geq 1$. Let

$$
D_{k}(K)=\operatorname{div}\left(M_{k}(K)\right) .
$$

For an affine element $K$, one has that

$$
\begin{aligned}
\operatorname{div}\left(B D M_{k}(K)\right) & =P_{k-1}(K) \\
\operatorname{div}\left(B D F M_{k+1}(K)\right) & =P_{k}(K) \\
\operatorname{div}\left(R T_{k}(K)\right) & =P_{k}(K), K \text { is a triangle/tetrahedron, } \\
\operatorname{div}\left(R T_{k}(K)\right) & =Q_{k}(K), K \text { is a rectangle/cube. }
\end{aligned}
$$

Starting from the local definitions, for each choice of $M_{k}(K)$, define the global $H($ div; $\Omega$ ) conforming space by

$$
M_{k}=\left\{\mathbf{v} \in H(\operatorname{div} ; \Omega)|\mathbf{v}|_{K} \in M_{k}(K), \quad \forall K \in \mathcal{T}_{h}\right\} .
$$

(See [10] for appropriate choices of degrees of freedom for these spaces.) $M_{k}$ will be replaced by $R T_{k}, B D M_{k}$, or $B D F M_{k+1}$ when specified spaces are needed. Similarly, define

$$
D_{k}=\left\{q \in L^{2}(\Omega)|q|_{K} \in D_{k}(K), \quad \forall K \in \mathcal{T}_{h}\right\}
$$

and denote the space of piecewise discontinuous polynomials of degree $\leq k$ by

$$
P_{k}=\left\{q \in L^{2}(\Omega)|q|_{K} \in P_{k}(K), \quad \forall K \in \mathcal{T}_{h}\right\} .
$$

Let $\mathcal{P}_{h}^{\prime}$ be the $L^{2}$ projection into $P_{k}$ and it is well known that

$$
\left\|q-\mathcal{P}_{h}^{\prime} q\right\|_{0, r} \leq C h^{s}\|q\|_{s, r} \quad 0 \leq s \leq k+1 .
$$

Let $\mathcal{P}_{h}$ be the $L^{2}$ projection into $D_{k}$. It is well known that for all $q \in H^{r}(\Omega)$,

$$
\left\|q-\mathcal{P}_{h} q\right\|_{0, r} \leq C h^{s}\|q\|_{s, r}
$$

where $s \leq k+1$ for $R T_{k}$ and $B D F M_{k+1}, s \leq k$ for $B D M_{k}$. It is also well known that there exists an interpolation operator $\Pi_{h}: H(\operatorname{div} ; \Omega) \cap L^{t}(\Omega)^{d} \rightarrow M_{k}$ for fixed $t>2$ satisfying the commutativity property

$$
\nabla \cdot\left(\Pi_{h} \mathbf{v}\right)=\mathcal{P}_{h} \nabla \cdot \mathbf{v} \quad \forall \mathbf{v} \in H(\operatorname{div} ; \Omega) \cap L^{t}(\Omega)^{d},
$$

and the approximation properties: for $2 \leq r \leq \infty$,

$$
\left\|\mathbf{v}-\Pi_{h} \mathbf{v}\right\|_{0, r} \leq C h^{s}\|\mathbf{v}\|_{s, r}
$$

where $\frac{1}{r}<s \leq k+1$ and

$$
\left\|\nabla \cdot\left(\mathbf{v}-\Pi_{h} \mathbf{v}\right)\right\|_{0,2} \leq C h^{s}\|\nabla \cdot \mathbf{v}\|_{s, 2},
$$

where $0 \leq s \leq k+1$ for $R T_{k}$ and $B D F M_{k+1}$ or $0 \leq s \leq k$ for $B D M_{k}$. 
Denote the product spaces by

$$
M_{k}^{d}=\prod_{i=1}^{d} M_{k} \quad \text { and } \quad D_{k}^{d}=\prod_{i=1}^{d} D_{k},
$$

define a subspace of $M_{k}^{d}$ by

$$
\hat{M}_{k}^{d}=\left\{\boldsymbol{\tau} \in M_{k}^{d} \mid \int_{\Omega} \operatorname{tr} \boldsymbol{\tau} d x=0\right\}
$$

and let

$$
X_{k}^{d}=\hat{M}_{k}^{d} \times P_{k} .
$$

Then our mixed finite element approximation is to find a triple $\left(\left(\boldsymbol{\sigma}_{h}, p_{h}\right), \mathbf{u}_{h}\right) \in$ $X_{k}^{d} \times D_{k}^{d}$ such that

$$
\begin{cases}a\left(\left(\boldsymbol{\sigma}_{h}, p_{h}\right),(\boldsymbol{\tau}, q)\right)+b\left((\boldsymbol{\tau}, q), \mathbf{u}_{h}\right)=g(\boldsymbol{\tau}) & \forall(\boldsymbol{\tau}, q) \in X_{k}^{d}, \\ b\left(\left(\boldsymbol{\sigma}_{h}, p_{h}\right), \mathbf{v}\right) & =f(\mathbf{v}) \quad \forall \mathbf{v} \in D_{k}^{d},\end{cases}
$$

where the bilinear forms $a(\cdot, \cdot)$ and $b(\cdot, \cdot)$ are defined as those in (2.5).

To establish well-posedness of (3.6) and error bounds, we define an interpolation operator $\boldsymbol{\Pi}_{h}: \widehat{H}(\operatorname{div} ; \Omega)^{d} \cap L^{t}(\Omega)^{d \times d} \rightarrow \hat{M}_{k}^{d}$ by

$$
\boldsymbol{\Pi}_{h} \boldsymbol{\tau}=\left(\Pi_{h} \boldsymbol{\tau}_{1}, \ldots, \Pi_{h} \boldsymbol{\tau}_{d}\right)-b \boldsymbol{\delta} \quad \text { with } \quad b=\frac{1}{d|\Omega|} \int_{\Omega} \operatorname{tr}\left(\Pi_{h} \boldsymbol{\tau}_{1}, \ldots, \Pi_{h} \boldsymbol{\tau}_{d}\right) d x
$$

and the $L^{2}$ projection operator into $D_{k}^{d}$ by

$$
\mathbf{P}_{h} \mathbf{v}=\left(\mathcal{P}_{h} v_{1}, \ldots, \mathcal{P}_{h} v_{d}\right) .
$$

By (3.3), (3.4), (3.5), and (3.2), it is then easy to check the validity of the commutativity property

$$
\nabla \cdot\left(\boldsymbol{\Pi}_{h} \boldsymbol{\tau}\right)=\mathbf{P}_{h} \nabla \cdot \boldsymbol{\tau} \quad \forall \boldsymbol{\tau} \in H(\operatorname{div} ; \Omega)^{d} \cap L^{t}(\Omega)^{d \times d},
$$

and the approximation properties: for $2 \leq r \leq \infty$,

$$
\left\|\mathbf{v}-\mathbf{P}_{h} \mathbf{v}\right\|_{0, r} \leq C h^{s}\|\mathbf{v}\|_{s, r},
$$

where $s \leq k+1$ for $R T_{k}$ and $B D F M_{k+1}, s \leq k$ for $B D M_{k}$,

$$
\left\|\boldsymbol{\tau}-\boldsymbol{\Pi}_{h} \boldsymbol{\tau}\right\|_{0, r} \leq C h^{s}\|\boldsymbol{\tau}\|_{s, r},
$$

where $\frac{1}{r} \leq s \leq k+1$, and

$$
\left\|\nabla \cdot\left(\boldsymbol{\tau}-\boldsymbol{\Pi}_{h} \boldsymbol{\tau}\right)\right\|_{0,2} \leq C h^{s}\|\nabla \cdot \boldsymbol{\tau}\|_{s, 2},
$$

where $0 \leq s \leq k$ for $B D M_{k}, 0 \leq s \leq k+1$ for $R T_{k}$ and $B D F M_{k+1}$.

Let

$$
Z_{k}^{d}=\left\{\boldsymbol{\tau} \in \hat{M}_{k}^{d} \mid(\nabla \cdot \boldsymbol{\tau}, \mathbf{v})=0 \quad \forall \mathbf{v} \in P_{k}^{d}\right\}
$$

and

$$
X_{k}^{0, d}=\left\{(\boldsymbol{\tau}, q) \in X_{k}^{d} \mid b((\boldsymbol{\tau}, q), \mathbf{v})=0 \quad \forall \mathbf{v} \in D_{k}^{d}\right\}=Z_{k}^{d} \times D_{k}
$$


The next two lemmas verify the coercivity of the bilinear form $a(\cdot, \cdot)$ in $X_{k}^{0, d}$ and the inf-sup condition of the bilinear form $b(\cdot, \cdot)$ in $X_{k}^{d} \times D_{k}^{d}$.

Lemma 3.1. There exists a positive constant $\hat{\alpha}$ independent of the mesh size $h$ such that

$$
\hat{\alpha}\|(\boldsymbol{\tau}, q)\|^{2} \leq a((\boldsymbol{\tau}, q),(\boldsymbol{\tau}, q))
$$

for any $(\boldsymbol{\tau}, q) \in X_{k}^{0, d}$.

Proof. The commutativity property in (3.7) gives that $\nabla \cdot \hat{M}_{k}^{d} \subset D_{k}^{d}$, which, in turn, implies that $Z_{k}^{d}$ is the divergence free subspace of $\hat{M}_{k}^{d}$. Hence, $X_{k}^{0, d} \subset X^{0, d}$. Now, the coercivity (3.11) follows from (2.10).

Lemma 3.2. There exists a positive constant $\hat{\beta}$ independent of the mesh size $h$ such that

$$
\sup _{(\boldsymbol{\tau}, q) \in X_{k}^{d}} \frac{b((\boldsymbol{\tau}, q) \mathbf{v})}{\|(\boldsymbol{\tau}, q)\|} \geq \hat{\beta}\|\mathbf{v}\|_{0,2}
$$

for any $\mathbf{v} \in D_{k}^{d}$

Proof. For any $\mathbf{v} \in D_{k}^{d}$, it is easy to see that

$$
\sup _{(\boldsymbol{\tau}, q) \in X_{k}^{d}} \frac{(\nabla \cdot \boldsymbol{\tau}, \mathbf{v})}{\|\mid(\boldsymbol{\tau}, q)\|} \geq \sup _{(\boldsymbol{\tau}, 0) \in X_{k}^{d}} \frac{(\nabla \cdot \boldsymbol{\tau}, \mathbf{v})}{\|\boldsymbol{\tau}\|_{H(\text { div })}} \geq \sup _{\boldsymbol{\tau} \in \hat{M}_{k}^{d}} \frac{(\nabla \cdot \boldsymbol{\tau}, \mathbf{v})}{\|\boldsymbol{\tau}\|_{H(\mathrm{div})}} .
$$

Now, to show the validity of (3.12), it suffices to prove that there exists a positive constant $\hat{\beta}$ independent of the mesh size $h$ such that for any $\mathbf{v}$ in $D_{k}^{d}$, we have

$$
\sup _{\boldsymbol{\tau} \in \hat{M}_{k}^{d}} \frac{(\nabla \cdot \boldsymbol{\tau}, \mathbf{v})}{\|\boldsymbol{\tau}\|_{H(\text { div })}} \geq \hat{\beta}\|\mathbf{v}\|_{0,2},
$$

which has been established in [15] for RT elements. In a similar fashion, we can prove the validity of (3.13) for both BDM and BDFM elements. This completes the proof of the lemma.

Now, we are ready to establish the well-posedness and error bounds of mixed finite element approximation.

Theorem 3.3. The discrete problem in (3.6) has a unique solution $\left(\left(\boldsymbol{\sigma}_{h}, p_{h}\right), \mathbf{u}_{h}\right)$ in $X_{k}^{d} \times D_{k}^{d}$. Let $(\boldsymbol{\sigma}, \mathbf{u})$ be the solution of (2.6), then we have

$$
\left\|(\boldsymbol{\sigma}, p)-\left(\boldsymbol{\sigma}_{h}, p_{h}\right)\right\| \leq C \inf _{(\boldsymbol{\tau}, q) \in X_{k}^{d}}\|(\boldsymbol{\sigma}, p)-(\boldsymbol{\tau}, q)\| \|
$$

and

$$
\left\|\mathbf{u}-\mathbf{u}_{h}\right\|_{0,2} \leq C\left(\inf _{\mathbf{v} \in D_{k}^{d}}\|\mathbf{u}-\mathbf{v}\|_{0,2}+\inf _{(\boldsymbol{\tau}, q) \in X_{k}^{d}}\|(\boldsymbol{\sigma}-\boldsymbol{\tau}, p-q)\|\right) .
$$

Moreover, assume that $\mathbf{f} \in H^{r}(\Omega)^{d}$ and $(\boldsymbol{\sigma}, p, \mathbf{u}) \in H^{r}(\Omega)^{d \times d} \times H^{r}(\Omega) \times H^{r}(\Omega)^{d}$. Then we have the error bounds

$$
\left\|(\boldsymbol{\sigma}, p)-\left(\boldsymbol{\sigma}_{h}, p_{h}\right)\right\| \leq \leq h^{r}\left(\|\boldsymbol{\sigma}\|_{r}+\|p\|_{r, 2}+\|\mathbf{f}\|_{r, 2}\right)
$$

and

$$
\left\|\mathbf{u}-\mathbf{u}_{h}\right\|_{0,2} \leq C h^{r}\left(\|\mathbf{u}\|_{r, 2}+\|\boldsymbol{\sigma}\|_{r, 2}+\|p\|_{r, 2}+\|\mathbf{f}\|_{r, 2}\right),
$$

where $r \leq k+1$ for $R T_{k}$ and $B D F M_{k+1}$ elements and $r \leq k$ for $B D M_{k}$ elements. 
Proof. Existence and uniqueness of problem (3.6) and error bounds in (3.14) and (3.15) follow from the abstract theory for the saddle-point problem (see, e.g., 6, 10]) and Lemmas 3.1 and 3.2 Since

$$
\inf _{(\boldsymbol{\tau}, q) \in X_{k}^{d}}\|(\boldsymbol{\sigma}, p)-(\boldsymbol{\tau}, q)\| \leq \inf _{\boldsymbol{\tau} \in \hat{M}_{k}^{d}}\|\boldsymbol{\sigma}-\boldsymbol{\tau}\|_{H(\text { div })}+\inf _{q \in P_{k}}\|p-q\|_{0,2}
$$

error bounds (3.16) and (3.17) follow from (3.14), (3.15), and the approximation properties in (3.1), (3.2), (3.9), (3.10), and (3.8).

The $L^{2}$ error estimates contained in (3.16) is optimal for both the $R T_{k}$ and $B D F M_{k+1}$ approximation, but not for the $B D M_{k}$ element.

Theorem 3.4. Let $\left(\left(\boldsymbol{\sigma}_{h}, p_{h}\right), \mathbf{u}_{h}\right)$ be the unique solution of (3.6) using BDM $M_{k}$ element with $k \geq 1$. Under the same assumption in Theorem [3.3, we have the optimal $L^{2}$ approximation

$$
\left\|\boldsymbol{\sigma}-\boldsymbol{\sigma}_{h}\right\|_{0,2}+\left\|p-p_{h}\right\|_{0,2} \leq C h^{r}\left(\|\boldsymbol{\sigma}\|_{r, 2}+\|p\|_{r, 2}+\|\mathbf{f}\|_{r, 2}\right)
$$

for all $r \leq k+1$.

Proof. For the $B D M_{k}$ elements with $k \geq 1$, we have

$$
M_{k}^{d}=B \hat{D} M_{k}^{d} \times P_{k} \quad \text { and } \quad D_{k}^{d}=P_{k-1}^{d} .
$$

Denote the discretization and the interpolation errors by

$$
\left(\mathbf{E}_{h}, e_{h}\right)=\left(\boldsymbol{\sigma}-\boldsymbol{\sigma}_{h}, p-p_{h}\right) \text { and }\left(\mathbf{E}_{I}, e_{I}\right)=\left(\boldsymbol{\sigma}-\boldsymbol{\Pi}_{h} \boldsymbol{\sigma}, p-P_{h}^{\prime} p\right),
$$

respectively, then the difference between (2.6) and (3.6) yields the following error equations:

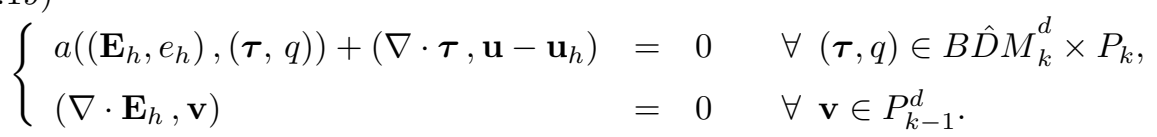

The commutativity property in (3.7) gives

$$
\left(\nabla \cdot \mathbf{E}_{I}, \mathbf{v}\right)=\left(\nabla \cdot\left(\boldsymbol{\sigma}-\Pi_{h} \boldsymbol{\sigma}\right), \mathbf{v}\right)=0 \quad \forall \mathbf{v} \in P_{k-1}^{d},
$$

which, together with the second equation in (3.19), leads to

$$
\nabla \cdot\left(\boldsymbol{\sigma}_{h}-\Pi_{h} \boldsymbol{\sigma}\right)=0 .
$$

To establish the optimal $L^{2}$ norm error bounds in (3.18), we use (2.11) with $(\boldsymbol{\tau}, q)=\left(\mathbf{E}_{h}, e_{h}\right)$ :

$$
\left\|\mathbf{E}_{h}\right\|_{0,2}^{2}+\left\|e_{h}\right\|_{0,2}^{2} \leq C\left(a\left(\left(\mathbf{E}_{h}, e_{h}\right),\left(\mathbf{E}_{h}, e_{h}\right)\right)+\left\|\nabla \cdot \mathbf{E}_{h}\right\|_{-1,2}^{2}\right) .
$$

By the first equation in (3.19), (3.21), and the Cauchy-Schwarz and triangle inequalities, we have

$$
\begin{aligned}
a\left(\left(\mathbf{E}_{h}, e_{h}\right),\left(\mathbf{E}_{h}, e_{h}\right)\right) & =a\left(\left(\mathbf{E}_{h}, e_{h}\right),\left(\mathbf{E}_{I}, e_{I}\right)\right)+a\left(\left(\mathbf{E}_{h}, e_{h}\right),\left(\Pi_{h} \boldsymbol{\sigma}-\boldsymbol{\sigma}_{h}, P_{h}^{\prime} p-p_{h}\right)\right) \\
& =a\left(\left(\mathbf{E}_{h}, e_{h}\right),\left(\mathbf{E}_{I}, e_{I}\right)\right)-\left(\nabla \cdot\left(\Pi_{h} \boldsymbol{\sigma}-\boldsymbol{\sigma}_{h}\right), \mathbf{u}-\mathbf{u}_{h}\right) \\
& =a\left(\left(\mathbf{E}_{h}, e_{h}\right),\left(\mathbf{E}_{I}, e_{I}\right)\right) \\
& \leq C \sqrt{a\left(\left(\mathbf{E}_{h}, e_{h}\right),\left(\mathbf{E}_{h}, e_{h}\right)\right)}\left(\left\|\mathbf{E}_{I}\right\|_{0,2}+\left\|e_{I}\right\|_{0,2}\right),
\end{aligned}
$$

which implies

$$
a\left(\left(\mathbf{E}_{h}, e_{h}\right),\left(\mathbf{E}_{h}, e_{h}\right)\right) \leq C\left(\left\|\mathbf{E}_{I}\right\|_{0,2}^{2}+\left\|e_{I}\right\|_{0,2}^{2}\right) .
$$


It follows from (3.21), the definition of the $H^{-1}$ norm, (3.20), the Cauchy-Schwarz inequality, and (3.8) with $r=1$ that

$$
\begin{aligned}
\left\|\nabla \cdot \mathbf{E}_{h}\right\|_{-1,2} & =\left\|\nabla \cdot \mathbf{E}_{I}\right\|_{-1,2}=\sup _{\mathbf{v} \in H_{0}^{1}(\Omega)^{d}} \frac{\left(\nabla \cdot \mathbf{E}_{I}, \mathbf{v}\right)}{\|\mathbf{v}\|_{1,2}}=\sup _{\mathbf{v} \in H_{0}^{1}(\Omega)^{d}} \frac{\left(\nabla \cdot \mathbf{E}_{I}, \mathbf{v}-\mathbf{P}_{h} \mathbf{v}\right)}{\|\mathbf{v}\|_{1,2}} \\
& \leq\left\|\nabla \cdot \mathbf{E}_{I}\right\|_{0,2} \sup _{\mathbf{v} \in H_{0}^{1}(\Omega)^{d}} \frac{\left\|\mathbf{v}-\mathbf{P}_{h} \mathbf{v}\right\|_{0,2}}{\|\mathbf{v}\|_{1,2}} \leq C h\left\|\nabla \cdot \mathbf{E}_{I}\right\|_{0,2} .
\end{aligned}
$$

Combining (3.22), (3.24), and (3.23) gives

$$
\left\|\mathbf{E}_{h}\right\|_{0,2}+\left\|e_{h}\right\|_{0,2} \leq h\left\|\nabla \cdot \mathbf{E}_{I}\right\|_{0,2}+\left\|\mathbf{E}_{I}\right\|_{0,2}+\left\|e_{I}\right\|_{0,2} .
$$

Now, (3.18) is a direct consequence of approximation properties in (3.1) with $q=p$ and (3.9) and (3.8) with $\boldsymbol{\tau}=\boldsymbol{\sigma}$. This completes the proof of the theorem.

Remark 3.5. For the pseudostress-velocity formulation in [15, similar results to those Theorems 3.3 and 3.4 can be proved for both the BDM and BDFM elements in a similar fashion. To ensure the optimal $L^{2}$ norm bound for the BDM element, the inequality in (2.7) from [14 is essential.

Remark 3.6. There are several approaches to numerically solve the algebraic system resulting in the mixed finite element methods for the Stokes equations. First, one can eliminate the velocity unknowns by the penalty method to obtain a symmetric, positive definite system on the pseudostress and the pressure unknowns, that may be numerically solved by fast and efficient $H$ (div) multigrid methods (see [3, 4, 26, 30]). This is similar to, but better than that for the pseudostress-velocity formulation [15, 17. Second, one can eliminate the pseudostress and the pressure unknowns to get a small discrete system involving only the Lagrange multiplier and the velocity through the hybridization technique (see, e.g., 22, 1]). Third, one can eliminate the Lagrange multiplier and the pseudostress by the hybridization technique and using appropriate quadrature rule to obtain finite volume discretizations on the velocity and the pressure (see 12 and Section 6). For the NavierStokes equations considered in the subsequent section, one can then combine the approaches mentioned above with various linearization procedures.

\section{The Stationary Navier-Stokes equations}

For the stationary Navier-Stokes equation, the analysis here follows our previous one on the pseudostress-velocity formulation in [16]. Introducing the pseudostress as in (2.1), the stationary Navier-Stokes equation may be rewritten in the following pseudostress-pressure-velocity formulation:

$$
\begin{cases}\frac{1}{\nu}(\boldsymbol{\sigma}+p \boldsymbol{\delta})-\nabla \mathbf{u} & =\mathbf{0} \\ \operatorname{tr} \boldsymbol{\sigma}+d p & =0 \\ \nabla \cdot \boldsymbol{\sigma}-\mathbf{u} \cdot \frac{1}{\nu}(\boldsymbol{\sigma}+p \boldsymbol{\delta}) & =-\mathbf{f} .\end{cases}
$$


Rescaling the stress, the pressure, and the right-hand side by $\boldsymbol{\sigma} / \nu \rightarrow \boldsymbol{\sigma}, p / \nu \rightarrow p$ and $\mathbf{f} / \nu \rightarrow \mathbf{f}$, respectively, system (4.1) may be written as

$$
\begin{cases}\boldsymbol{\sigma}+p \boldsymbol{\delta}-\nabla \mathbf{u} & =\mathbf{0}, \\ \operatorname{tr} \boldsymbol{\sigma}+d p & =0, \\ \nabla \cdot \boldsymbol{\sigma}-\frac{1}{\nu} c(\boldsymbol{\sigma}, p, \mathbf{u}) & =-\mathbf{f} .\end{cases}
$$

where $c(\boldsymbol{\sigma}, p, \mathbf{u})=\mathbf{u} \cdot(\boldsymbol{\sigma}+p \boldsymbol{\delta})-\nabla \nu \cdot \boldsymbol{\sigma}$. For simplicity of presentation, we assume that the viscosity parameter is a positive constant in this paper so that

$$
c(\boldsymbol{\sigma}, p, \mathbf{u})=\mathbf{u} \cdot(\boldsymbol{\sigma}+p \boldsymbol{\delta}) .
$$

The corresponding variational form of (4.2) and (1.2) is to find

$$
(\boldsymbol{\sigma}, p, \mathbf{u}) \in \widehat{W}^{0,3}(\operatorname{div} ; \Omega)^{d} \times L^{3}(\Omega)^{d} \times L^{2}(\Omega)^{d}
$$

such that

$$
\begin{cases}(\boldsymbol{\sigma}+p \boldsymbol{\delta}, \boldsymbol{\tau}+q \boldsymbol{\delta})+(\nabla \cdot \boldsymbol{\tau}, \mathbf{u})=g(\boldsymbol{\tau}) & \forall(\boldsymbol{\tau}, q) \in \widehat{H}(\operatorname{div} ; \Omega)^{d} \times L^{2}(\Omega), \\ (\nabla \cdot \boldsymbol{\sigma}, \mathbf{v})-\frac{1}{\nu}(\mathbf{u} \cdot(\boldsymbol{\sigma}+p \boldsymbol{\delta}), \mathbf{v})=f(\mathbf{v}) & \forall \mathbf{v} \in L^{2}(\Omega)^{d}\end{cases}
$$

Remark 4.1. The variational form in (4.3) uses smaller trial spaces $\widehat{W}^{0,3}(\operatorname{div} ; \Omega)^{d} \subset$ $\widehat{H}(\operatorname{div} ; \Omega)^{d}$ and $L^{3}(\Omega) \subset L^{2}(\Omega)$ than the Stokes equations for the pseudostress and the pressure, respectively. By doing so, we can guarantee the nonlinear term, $\mathbf{u} \cdot(\boldsymbol{\sigma}+p \boldsymbol{\delta})$, being in $L^{2}(\Omega)^{d}$ and, hence, the second equation in (4.3) is well defined. To see why, note first that the first equation in (4.3) implies that $\mathbf{u} \in H^{1}(\Omega)$; see Lemma 2.3 of [16]. Since the imbedding theorem implies that $H^{1}(\Omega)$ is continuously imbedded in $L^{r}(\Omega)$ with $r \in[1, \infty)$ for $d=2$ and $r \in[1,6]$ for $d=3$, it then follows from the Hölder inequality with $p=3$ and $q=3 / 2$ that

$$
\|\mathbf{u} \cdot(\boldsymbol{\sigma}+p \boldsymbol{\delta})\|_{0,2} \leq C\|\mathbf{u}\|_{0,6}\left(\|\boldsymbol{\sigma}\|_{0,3}+\|p\|_{0,3}\right) \leq C\|\mathbf{u}\|_{1,2}\left(\|\boldsymbol{\sigma}\|_{0,3}+\|p\|_{0,3}\right) .
$$

In the velocity-pressure formulation of the stationary Navier-Stokes equation with constant viscosity, rescaling the pressure and the right-hand side by $p / \nu \rightarrow p$ and $\mathbf{f} / \nu \rightarrow \mathbf{f}$, respectively, gives

$$
\begin{cases}\frac{1}{\nu} \mathbf{u} \cdot \nabla \mathbf{u}-\Delta \mathbf{u}+\nabla p & =\mathbf{f} \\ \nabla \cdot \mathbf{u} & =0\end{cases}
$$

Let

$$
H_{\mathbf{g}}^{1}(\Omega)^{d}=\left\{\mathbf{u} \in H^{1}(\Omega)^{d}|\mathbf{u}|_{\partial \Omega}=\mathbf{g}\right\} .
$$

The variational form of (4.5) and (1.2) is to find $(\mathbf{u}, p) \in H_{\mathbf{g}}^{1}(\Omega)^{d} \times L_{0}^{2}(\Omega)$ such that

$$
\left\{\begin{array}{llll}
(\nabla \mathbf{u}, \nabla \mathbf{v})+\frac{1}{\nu}(\mathbf{u} \cdot \nabla \mathbf{u}, \mathbf{v})-(p, \nabla \cdot \mathbf{v}) & =-f(\mathbf{v}) & & \forall \mathbf{v} \in H_{0}^{1}(\Omega)^{d}, \\
(\nabla \cdot \mathbf{u}, q) & =0 & \forall q \in L_{0}^{2}(\Omega) .
\end{array}\right.
$$

Under the assumption on the boundary in Section 1.1, the following theorem is well known (see, e.g., [18, 24]). 
Theorem 4.2. For $\mathbf{f} \in H^{l-1}(\Omega)^{d}$ and $\mathbf{g} \in H^{l+1 / 2}(\partial \Omega)^{d}$ with $l=0,1$, system (4.6) has solutions, $(\mathbf{u}, p)$, belonging to $H^{l+1}(\Omega)^{d} \times H^{l}(\Omega)$. Moreover, if $\nu>\nu_{0}(\Omega ; \mathbf{f}, \mathbf{g})^{1}$, then the solution $(\mathbf{u}, p)$ is unique.

In the rest of this section, we establish the well-posedness and uniqueness of system (4.3).

Lemma 4.3. For given $\mathbf{f} \in L^{2}(\Omega)^{d}$ and $\mathbf{g} \in H^{1 / 2}(\partial \Omega)^{d}$, let

$$
\left(\boldsymbol{\sigma}^{*}, p^{*}, \mathbf{u}^{*}\right) \in \widehat{W}^{0,3}(\operatorname{div} ; \Omega)^{d} \times L^{3}(\Omega) \times L^{2}(\Omega)^{d}
$$

be a solution of (4.3), then $\left(\mathbf{u}^{*}, p^{*}\right)$ satisfies (4.6).

Proof. By a standard argument, the first equation in (4.3) implies that

$$
\nabla \mathbf{u}^{*}=\boldsymbol{\sigma}^{*}+p^{*} \boldsymbol{\delta} \text { and } p^{*}=-\frac{1}{d} \operatorname{tr} \boldsymbol{\sigma}^{*} \text { in } \Omega
$$

and that $\mathbf{u}^{*}=\mathbf{g}$ on $\partial \Omega$. In (4.7), taking the trace of the first equation and using the second equation gives

$$
\nabla \cdot \mathbf{u}^{*}=0 \text { in } \Omega
$$

which yields that $\left(\mathbf{u}^{*}, p^{*}\right)$ satisfies the second equation in (4.6). The second equation in (4.7) and the fact that $\left(\boldsymbol{\sigma}^{*}, p^{*}, \mathbf{u}^{*}\right) \in \widehat{W}^{0,3}(\operatorname{div} ; \Omega)^{d} \times L^{3}(\Omega) \times L^{2}(\Omega)^{d}$ implies $p^{*} \in L_{0}^{2}(\Omega)$. Finally, substituting (4.7) into the second equation in (4.3) shows that $\left(\mathbf{u}^{*}, p^{*}\right)$ satisfies the first equation in (4.6). This completes the proof of the lemma.

Lemma 4.4. For given $\mathbf{f} \in L^{2}(\Omega)^{d}$ and $\mathbf{g} \in H^{1 / 2+\gamma}(\partial \Omega)^{d}$ with $\frac{1}{2} \leq \gamma \leq 1$, let $\left(\mathbf{u}^{\dagger}, p^{\dagger}\right) \in\left(H^{1+\gamma}(\Omega)^{d} \cap H_{\mathbf{g}}^{1}(\Omega)^{d}\right) \times\left(H^{\gamma}(\Omega) / \mathbb{R}\right)$ be a solution of (4.6), then $\left(\nabla \mathbf{u}^{\dagger}-\right.$ $p^{\dagger} \boldsymbol{\delta}, p^{\dagger}, \mathbf{u}^{\dagger}$ ) satisfies (4.3).

Proof. Let $\left(\mathbf{u}^{\dagger}, p^{\dagger}\right)$ be the solution of (4.6) and let $\boldsymbol{\sigma}^{\dagger}=\nabla \mathbf{u}^{\dagger}-p^{\dagger} \boldsymbol{\delta}$, then the first equation in (4.5) gives

$$
\nabla \cdot \boldsymbol{\sigma}^{\dagger}=\frac{1}{\nu} \mathbf{u}^{\dagger} \cdot \nabla \mathbf{u}^{\dagger}-\mathbf{f} .
$$

The smoothness of $\left(\mathbf{u}^{\dagger}, p^{\dagger}\right)$ implies that $\boldsymbol{\sigma}^{\dagger}$ lies in $L^{3}(\Omega)^{d \times d}$ and that the above equation holds in the $L^{2}$ sense. It is then straightforward to see that $\left(\boldsymbol{\sigma}^{\dagger}, p^{\dagger}, \mathbf{u}^{\dagger}\right)=$ $\left(\nabla \mathbf{u}^{\dagger}-p^{\dagger} \boldsymbol{\delta}, p^{\dagger}, \mathbf{u}^{\dagger}\right)$ is a solution of (4.3).

Theorem 4.5. For given $\mathbf{f} \in L^{2}(\Omega)^{d}$ and $\mathbf{g} \in H^{1 / 2+\gamma}(\partial \Omega)^{d}$ with $\frac{1}{2} \leq \gamma \leq 1$, system (4.3) has a solution, $(\boldsymbol{\sigma}, p, \mathbf{u})$, belonging to $H^{\gamma}(\Omega)^{d \times d} \times H^{\gamma}(\Omega) \times H^{1+\gamma}(\Omega)^{d}$. Moreover, if $\nu>\nu_{0}(\Omega ; \mathbf{f}, \mathbf{g})$, then the solution $(\boldsymbol{\sigma}, p, \mathbf{u})$ is unique.

Proof. The theorem is a direct consequence of Theorem 4.2 and Lemmas 4.3 and 4.4 .

$$
\begin{aligned}
& { }^{1} \text { Here } \nu_{0}(\Omega ; \mathbf{f}, \mathbf{g}) \text { is defined by } \\
& \qquad \nu_{0}=\inf \left\{\rho\left(\mathbf{u}_{0}\right)+\left(\mathcal{N}\left\|l\left(\mathbf{f} ; \mathbf{u}_{0}\right)\right\|_{V^{\prime}}\right)^{1 / 2} \mid \mathbf{u}_{0} \in H_{\mathbf{g}}^{1}(\Omega)^{d} \text { and } \nabla \cdot \mathbf{u}_{0}=0\right\} \\
& \text { with } \\
& V=\left\{\mathbf{v} \in H_{0}^{1}(\Omega)^{d} \mid \nabla \cdot \mathbf{u}_{0}=0\right\}, \quad \rho\left(\mathbf{u}_{0}\right)=\sup _{\mathbf{v} \in V} \frac{\left(\mathbf{v} \cdot \nabla \mathbf{u}_{0}, \mathbf{v}\right)}{|\mathbf{v}|_{1, \Omega}^{2}}, \\
& \mathcal{N}=\sup _{\mathbf{u}, \mathbf{v}, \mathbf{w} \in V} \frac{(\mathbf{w} \cdot \nabla \mathbf{u}, \mathbf{v})}{|\mathbf{u}|_{1, \Omega}|\mathbf{v}|_{1, \Omega}|\mathbf{w}|_{1, \Omega}}, \quad\left\|l\left(\mathbf{f} ; \mathbf{u}_{0}\right)\right\|_{V^{\prime}}=\sup _{\mathbf{v} \in V} \frac{\nu(\mathbf{f}, \mathbf{v})-\nu\left(\nabla \mathbf{u}_{0}, \nabla \mathbf{u}_{0}\right)-\left(\mathbf{u}_{0} \cdot \nabla \mathbf{u}_{0}, \mathbf{v}\right)}{|\mathbf{v}|_{1, \Omega}} .
\end{aligned}
$$


Our mixed finite element approximation is to find a pair $\left(\boldsymbol{\sigma}_{h}, p_{h}, \mathbf{u}_{h}\right) \in \hat{M}_{k}^{d} \times$ $P_{k} \times D_{k}^{d}$ such that

$$
\begin{cases}\left(\boldsymbol{\sigma}_{h}+p_{h} \boldsymbol{\delta}, \boldsymbol{\tau}+q \boldsymbol{\delta}\right)+\left(\nabla \cdot \boldsymbol{\tau}, \mathbf{u}_{h}\right)=g(\boldsymbol{\tau}) & \forall \boldsymbol{\tau} \in \hat{M}_{k}^{d} \times P_{k}, \\ \left(\nabla \cdot \boldsymbol{\sigma}_{h}, \mathbf{v}\right)-\frac{1}{\nu}\left(\mathbf{u}_{h} \cdot\left(\boldsymbol{\sigma}_{h}+p_{h} \boldsymbol{\delta}\right), \mathbf{v}\right)=f(\mathbf{v}) & \forall \mathbf{v} \in D_{k}^{d} .\end{cases}
$$

\section{Convergence analysis of Navier-Stokes equations}

To analyze the convergence of the finite element approximation, we make use of the abstract approximation theory of Brezzi, Rappaz, and Raviart [11, 24]. For a concise presentation of the main theory, see Section 3.3 (Theorem 3.3 and Remark 3.4) in Chapter IV of [24] or Section 4 of [16].

To apply the abstract theory, similar to the velocity-pressure formulation, it is natural to choose

$$
G(\nu, \phi)=\left(-\mathbf{g}, \frac{1}{\nu} \mathbf{u} \cdot(\boldsymbol{\sigma}+p \boldsymbol{\delta})-\mathbf{f}\right)
$$

for $\phi=(\boldsymbol{\sigma}, p, \mathbf{u})$, which contains the nonlinear term and the given data. Since the momentum equation is required to be valid in $L^{2}(\Omega)^{d}$ (see (4.3) ), we set

$$
\mathbf{Y}=H^{\frac{3}{2}}(\partial \Omega)^{d} \times L^{2}(\Omega)^{d}
$$

and let $\mathbf{Z}=\mathbf{Y}$. Here we assumed $\mathbf{g} \in H^{\frac{3}{2}}(\partial \Omega)^{d}$ to guarantee the exact solution $(\boldsymbol{\sigma}, p, \mathbf{u})$ is in $H^{1}(\Omega)^{d \times d} \times H^{1}(\Omega) \times H^{2}(\Omega)^{d}$.

A simple calculation gives that the Fréchet derivative of the operator $G$ with respect to $\phi$ is

$$
D_{\phi} G(\nu, \phi)[\psi]=\left(\mathbf{0}, \frac{1}{\nu}(\mathbf{u} \cdot(\boldsymbol{\tau}+q \boldsymbol{\delta})+\mathbf{v} \cdot(\boldsymbol{\sigma}+p \boldsymbol{\delta}))\right)
$$

for $\psi=(\boldsymbol{\tau}, q, \mathbf{v})$.

Let

$$
\mathbf{X}=L^{3}(\Omega)^{d \times d} \times L^{3}(\Omega) \times L^{3}(\Omega)^{d}
$$

with the norm

$$
\|(\boldsymbol{\tau}, q, \mathbf{v})\|_{\mathbf{X}}=\left(\|\boldsymbol{\tau}\|_{0,3}^{2}+\|q\|_{0,3}^{2}+\|\mathbf{v}\|_{0,3}^{2}\right)^{\frac{1}{2}} .
$$

This choice of $\mathbf{X}$ yields $D_{\phi} G(\nu, \phi)[\psi]$ defined in (5.2) belonging to $\mathbf{Z}$ for any $\psi \in \mathbf{X}$ if $\phi=(\boldsymbol{\sigma}, p, \mathbf{u})$ is the exact solution of (4.3) and $(\boldsymbol{\sigma}, p, \mathbf{u})$ is in $H^{1}(\Omega)^{d \times d} \times H^{1}(\Omega) \times$ $H^{2}(\Omega)^{d}$.

In the remainder of this section, we define the Stokes solution operator and estimate the discretization error in the $\|\cdot\|_{\mathbf{X}}$ norm needed in the abstract theory in subsection 5.1. Subsection 5.2 proves that a unique solution of (4.3) is also a branch of nonsingular solution. Finally, error estimates for the Navier-Stokes equation are established in subsection 5.3. 
5.1. The Stokes solution operators. For any $\left(\mathbf{g}_{*}, \mathbf{f}_{*}\right) \in \mathbf{Y}$, define $T: \mathbf{Y} \rightarrow \mathbf{X}$ by

$$
T\left(\mathbf{g}_{*}, \mathbf{f}_{*}\right)=(\boldsymbol{\sigma}, p, \mathbf{u}) \in \mathbf{X},
$$

where $(\boldsymbol{\sigma}, p, \mathbf{u})$ is the solution of the Stokes equations in the pseudostress-velocity formulation

$$
\begin{cases}(\boldsymbol{\sigma}+p \boldsymbol{\delta}, \boldsymbol{\tau}+q \boldsymbol{\delta})+(\mathbf{u}, \nabla \cdot \boldsymbol{\tau})=g_{*}(\boldsymbol{\tau}) & \forall(\boldsymbol{\tau}, q) \in \widehat{H}(\operatorname{div} ; \Omega)^{d} \times L^{2}(\Omega) \\ (\nabla \cdot \boldsymbol{\sigma}, \mathbf{v})=f_{*}(\mathbf{v}) & \forall \mathbf{v} \in L^{2}(\Omega)^{d}\end{cases}
$$

Here the linear forms are defined by

$$
g_{*}(\boldsymbol{\tau})=\int_{\partial \Omega}(\mathbf{n} \cdot \boldsymbol{\tau}) \cdot \mathbf{g}_{*} \mathrm{~d} s \quad \text { and } \quad f_{*}(\mathbf{v})=-\int_{\Omega} \mathbf{f}_{*} \cdot \mathbf{v} \mathrm{d} \mathbf{x} .
$$

Lemma 5.1. For any $\left(\mathbf{g}_{*}, \mathbf{f}_{*}\right) \in \mathbf{Y}$, problem (5.4) has a unique solution $(\boldsymbol{\sigma}, p, \mathbf{u})=$ $T\left(\mathbf{g}_{*}, \mathbf{f}_{*}\right)$ which is in $H^{1}(\Omega)^{d \times d} \times H^{1}(\Omega) \times H^{2}(\Omega)^{d} \subset \mathbf{X}$.

Proof. It is shown in Theorem 2.4 that problem (5.4) has a unique solution $(\boldsymbol{\sigma}, p, \mathbf{u})$ $\in \widehat{H}(\operatorname{div} ; \Omega)^{d} \times L^{2}(\Omega) \times L^{2}(\Omega)^{d}$. A similar argument as that of Lemma 4.3 yields that $(\mathbf{u}, p) \in H_{\mathbf{g}_{*}}^{1}(\Omega)^{d} \times L_{0}^{2}(\Omega)$ satisfies the corresponding Stokes equation and that

$$
\boldsymbol{\sigma}=-p \boldsymbol{\delta}+\nabla \mathbf{u} \text { and } \operatorname{tr} \boldsymbol{\sigma}+d p=0 .
$$

Now, the $H^{2}$ full regularity, $(\mathbf{u}, p) \in H^{2}(\Omega)^{d} \times H^{1}(\Omega)$, (see, e.g., 24]) of the stationary Stokes equation implies that $(\boldsymbol{\sigma}, p, \mathbf{u}) \in H^{1}(\Omega)^{d \times d} \times H^{1}(\Omega) \times H^{2}(\Omega)^{d} \subset \mathbf{X}$.

Denote by

$$
\mathbf{X}_{h}=\hat{M}_{k}^{d} \times P_{k} \times D_{k}^{d} \subset \mathbf{X}
$$

the finite element space and define $T_{h}: \mathbf{Y} \rightarrow \mathbf{X}_{h}$ by

$$
T_{h}\left(\mathbf{g}_{*}, \mathbf{f}_{*}\right)=\left(\boldsymbol{\sigma}_{h}, p_{h}, \mathbf{u}_{h}\right) \in \mathbf{X}_{h},
$$

where $\left(\boldsymbol{\sigma}_{h}, p_{h}, \mathbf{u}_{h}\right)$ is the solution of the discrete counterpart of (5.4)

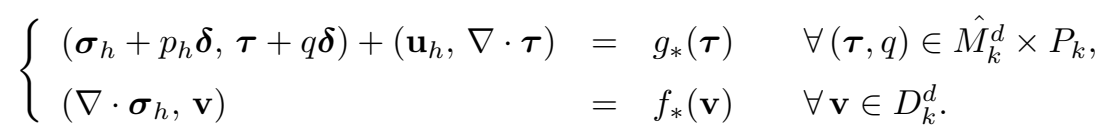

The well-posedness, the a priori estimate, and the standard $H(\operatorname{div}) \times L_{0}^{2} \times L^{2}$ error estimates have been done in the previous sections.

With $\mathbf{Z}=\mathbf{Y}$, the following condition $((3.38)$ on p. 306 of [24] or (4.10) of [16])

$$
\lim _{h \rightarrow 0}\left\|T-T_{h}\right\|_{\mathcal{L}(\mathbf{Z}, \mathbf{X})}=0
$$

implies $((3.37)$ on p. 306 of [24] or (4.9) of [16])

$$
\lim _{h \rightarrow 0}\left\|\left(T-T_{h}\right) \mathbf{g}\right\|_{\mathbf{X}}=0 \quad \forall \mathbf{g} \in \mathbf{Y} .
$$

The next theorem shows that the operators $T$ and $T_{h}$ satisfy condition (5.8) and estimates the error bound in the $\|\cdot\|_{\mathbf{x}}$ norm. 
Theorem 5.2. For any $\left(\mathbf{g}_{*}, \mathbf{f}_{*}\right) \in \mathbf{Y}$, let $(\boldsymbol{\sigma}, p, \mathbf{u})=T\left(\mathbf{g}_{*}, \mathbf{f}_{*}\right)$ and $\left(\boldsymbol{\sigma}_{h}, p_{h}, \mathbf{u}_{h}\right)=$ $T_{h}\left(\mathbf{g}_{*}, \mathbf{f}_{*}\right)$ be the solutions of (5.4) and (5.6), respectively. Then

$$
\lim _{h \rightarrow 0}\left\|T-T_{h}\right\|_{\mathcal{L}(\mathbf{Z}, \mathbf{X})}=0 .
$$

Moreover, assume that $(\boldsymbol{\sigma}, p, \mathbf{u}) \in H^{r}(\Omega)^{d \times d} \times H^{r}(\Omega)^{d} \times H^{r}(\Omega)^{d}$ for $1 \leq r \leq k+1$, then there exists a positive constant $C$ independent of $h$ such that

$$
\left\|T\left(\mathbf{g}_{*}, \mathbf{f}_{*}\right)-T_{h}\left(\mathbf{g}_{*}, \mathbf{f}_{*}\right)\right\|_{\mathbf{X}} \leq C h^{r-\alpha}\left(\|\boldsymbol{\sigma}\|_{r, 2}+\|p\|_{r, 2}+\|\mathbf{u}\|_{r, 2}\right)
$$

with $\alpha=d / 6$.

Proof. The theorem can be proved by using arguments similar to the proof of Theorem 5.2 in [16] for the pseudostress-velocity formulation.

5.2. A branch of nonsingular solutions. Let $\Lambda \subset(0, \infty)$ be a compact interval. Given $(\mathbf{g}, \mathbf{f}) \in \mathbf{Y}$, for any $(\nu, \phi)=(\nu,(\boldsymbol{\sigma}, p, \mathbf{u})) \in \Lambda \times \mathbf{X}$, define $F: \Lambda \times \mathbf{X} \rightarrow \mathbf{X}$ by

$$
F(\nu, \phi)=\phi+T G(\nu, \phi) .
$$

Consider the following nonlinear problem: find $(\nu, \phi(\nu)) \in \Lambda \times \mathbf{X}$ such that

$$
F(\nu, \phi)=0 .
$$

The set $\{(\nu, \phi(\nu)) \mid \nu \in \Lambda\}$ is called a branch of solutions of (5.12) if $F(\nu, \phi(\nu))=0$ for $\nu \in \Lambda$ and the map $\nu \mapsto \phi(\nu)$ is a continuous function from $\Lambda$ into $\mathbf{X}$. If, in addition, the Fréchet derivative $D_{\phi} F(\nu, \phi(\nu))$ of $F$ with respect to $\phi$ is an isomorphism from $\mathbf{X}$ onto $\mathbf{X}$ for all $\nu \in \Lambda$, then the branch of solutions $\{(\nu, \phi(\nu)) \mid \nu \in \Lambda\}$ is called nonsingular. Approximations of (5.12) are to find $\left(\nu, \phi_{h}(\nu)\right) \in \Lambda \times \mathbf{X}_{h}$ such that

$$
F_{h}\left(\nu, \phi_{h}\right) \equiv \phi_{h}+T_{h} G\left(\nu, \phi_{h}\right)=0 .
$$

It is easy to see, by (5.2), that the Fréchet derivative of the operator $F$ with respect to $\phi$ is

$$
D_{\phi} F(\nu, \phi)[\psi]=\psi+T D_{\phi} G(\nu, \phi)[\psi]=\psi+T\left(\mathbf{0}, \frac{1}{\nu}(\mathbf{u} \cdot(\boldsymbol{\tau}+q \boldsymbol{\delta})+\mathbf{v} \cdot(\boldsymbol{\sigma}+p \boldsymbol{\sigma}))\right)
$$

for any $\psi=(\boldsymbol{\tau}, \mathbf{v}) \in \mathbf{X}$.

Lemma 5.3. Let $(\mathbf{g}, \mathbf{f}) \in \mathbf{Y}$. Then $(\boldsymbol{\sigma}, p, \mathbf{u}) \in \mathbf{X}$ is a solution of (4.3) if and only if $(\nu, \phi)=(\nu,(\boldsymbol{\sigma}, p, \mathbf{u}))$ is a solution of (5.12) with the operators $T$ and $G$ defined in the respective (5.3) and (5.1).

Proof. Rewrite problem (4.3) as follows:

$$
\begin{cases}(\boldsymbol{\sigma}+p \boldsymbol{\delta}, \boldsymbol{\tau}+q \boldsymbol{\delta})+(\nabla \cdot \boldsymbol{\tau}, \mathbf{u})=g(\boldsymbol{\tau}) & \forall(\boldsymbol{\tau}, q) \in \widehat{H}(\operatorname{div} ; \Omega)^{d} \times L^{2}(\Omega), \\ (\nabla \cdot \boldsymbol{\sigma}, \mathbf{v})=\left(\frac{1}{\nu} \mathbf{u} \cdot(\boldsymbol{\sigma}+p \boldsymbol{\delta})-\mathbf{f}, \mathbf{v}\right) & \forall \mathbf{v} \in L^{2}(\Omega)^{d}\end{cases}
$$


By the definitions of the operators of $T$ in the previous section and $G$ in (5.1), it is equivalent to

$$
\phi=(\boldsymbol{\sigma}, p, \mathbf{u})=-T\left(-\mathbf{g}, \frac{1}{\nu} \mathbf{u} \cdot(\boldsymbol{\sigma}+p \boldsymbol{\delta})-\mathbf{f}\right)=-T G(\nu, \phi) .
$$

That is, $F(\nu, \phi)=0$.

Remark 5.4. Similarly, $\left(\boldsymbol{\sigma}_{h}, p_{h}, \mathbf{u}_{h}\right)$ is a solution of (4.8) if and only if $\left(\nu, \phi_{h}\right)=$ $\left(\nu,\left(\boldsymbol{\sigma}_{h}, \mathbf{u}_{h}\right)\right)$ is a solution of (5.13) with the operator $T_{h}$ defined in (5.5).

Theorem 5.5. For $(\mathbf{g}, \mathbf{f}) \in \mathbf{Y}$, assume that $\nu>\nu_{0}(\Omega ; \mathbf{f}, \mathbf{g})$. Then (5.12), with the operators $T$ and $G$ defined in the respective (5.3) and (5.1), has a branch of nonsingular solutions.

Proof. Let $(\boldsymbol{\sigma}, p, \mathbf{u})$ be the unique solution of (4.3), by Lemma 5.3. $(\nu, \phi)=$ $(\nu,(\boldsymbol{\sigma}, p, \mathbf{u}))$ is a branch solution of (5.12). To prove that $(\nu, \phi)$ is nonsingular, i.e., $D_{\phi} F(\nu, \phi)$ is an isomorphism from $\mathbf{X}$ onto $\mathbf{X}$, it suffices to show that for every $\omega=(\boldsymbol{\zeta}, s, \mathbf{w}) \in \mathbf{X}$, there exists a unique $\psi^{\prime}=\left(\boldsymbol{\tau}^{\prime}, q^{\prime}, \mathbf{v}^{\prime}\right) \in \mathbf{X}$ such that $D_{\phi} F(\nu, \phi)\left[\psi^{\prime}\right]=\omega$. By (5.14), this becomes

$$
\omega-\psi^{\prime}=T\left(\mathbf{0}, \frac{1}{\nu}\left(\mathbf{u} \cdot\left(\boldsymbol{\tau}^{\prime}+q^{\prime} \boldsymbol{\delta}\right)+\mathbf{v}^{\prime} \cdot(\boldsymbol{\sigma}+p \boldsymbol{\delta})\right)\right) .
$$

By the definition of $T$, this is equivalent to finding a unique $\psi=(\boldsymbol{\tau}, q, \mathbf{v})=$ $\left(\boldsymbol{\zeta}-\boldsymbol{\tau}^{\prime}, s-q^{\prime}, \mathbf{w}-\mathbf{v}^{\prime}\right) \in \mathbf{X}$ such that

$$
\left\{\begin{array}{l}
(\boldsymbol{\tau}+q \boldsymbol{\delta}, \boldsymbol{\xi}+t \boldsymbol{\delta})+(\nabla \cdot \boldsymbol{\xi}, \mathbf{v})=0 \quad \forall(\boldsymbol{\xi}, t) \in \widehat{H}(\operatorname{div} ; \Omega)^{d} \times L^{2}(\Omega), \\
(\nabla \cdot \boldsymbol{\tau}, \mathbf{z})-\frac{1}{\nu}(\mathbf{u} \cdot(\boldsymbol{\tau}+q \boldsymbol{\delta})+\mathbf{v} \cdot(\boldsymbol{\sigma}+p \boldsymbol{\delta}), \mathbf{z})=-\frac{1}{\nu}\left(\mathbf{f}_{*}, \mathbf{z}\right) \quad \forall \mathbf{z} \in L^{2}(\Omega)^{d},
\end{array}\right.
$$

where $\mathbf{f}_{*}=\mathbf{u} \cdot(\boldsymbol{\zeta}+s \boldsymbol{\delta})+\mathbf{w} \cdot(\boldsymbol{\sigma}+p \boldsymbol{\delta})$. It is easy to check that the corresponding velocity-pressure formulation of (5.15) is to find $(\mathbf{v}, t) \in H_{0}^{1}(\Omega)^{d} \times L_{0}^{2}(\Omega)$ such that (5.16)

$$
\begin{cases}(\nabla \mathbf{v}, \nabla \mathbf{z})-(t, \nabla \cdot \mathbf{z})+\frac{1}{\nu}(\mathbf{u} \cdot \nabla \mathbf{v}+\mathbf{v} \cdot \nabla \mathbf{u}, \mathbf{z})=\frac{1}{\nu}\left(\mathbf{f}_{*}, \mathbf{z}\right) & \forall \mathbf{z} \in H_{0}^{1}(\Omega)^{d}, \\ (\nabla \cdot \mathbf{v}, r)=0 & \forall r \in L_{0}^{2}(\Omega) .\end{cases}
$$

Under the assumption that $\nu>\nu_{0}(\Omega ; \mathbf{f}, \mathbf{g})$, it is proved in Chapter IV of 24] that (5.16) has a unique solution $(\mathbf{v}, t) \in H_{0}^{1}(\Omega)^{d} \times L_{0}^{2}(\Omega)$. Since $\mathbf{u} \in H^{2}(\Omega)^{d}$, then $\mathbf{f}_{*}-\mathbf{u} \cdot \nabla \mathbf{v}-\mathbf{v} \cdot \nabla \mathbf{u}$ belongs to $L^{2}(\Omega)^{d}$. By the $H^{2}$ regularity of the Stokes equation, we have that $(\mathbf{v}, t)$ is in $H^{2}(\Omega)^{d} \times H^{1}(\Omega)$. Now, a similar argument as that of Lemma 4.4 shows that (5.15) has a unique solution and, hence, $(\nu, \phi)$ is a branch of nonsingular solutions.

\subsection{Error estimates.}

Theorem 5.6. Let $(\mathbf{f}, \mathbf{g}) \in \mathbf{Y}$. Assume that $\nu>\nu_{0}(\Omega ; \mathbf{f}, \mathbf{g})$ and that $(\nu, \phi(\nu))=$ $(\nu,(\boldsymbol{\sigma}(\nu), p(\nu), \mathbf{u}(\nu)))$ is a branch of nonsingular solutions of (5.12). Then for $h$ sufficiently small, there exist a neighborhood $\mathcal{O}$ of the origin in $\mathbf{X}$ and a unique $\mathscr{C}^{2}$ function $\nu \rightarrow \phi_{h} \in \mathbf{X}_{h}$ such that $\left\{\left(\nu, \phi_{h}(\nu)\right) \mid \nu \in \Lambda\right\}$ is a branch of nonsingular solutions of (5.13) and that $\phi(\nu)-\phi_{h}(\nu) \in \mathcal{O}$ for all $\nu \in \Lambda$. 
Moreover, assume that $(\boldsymbol{\sigma}(\nu), p(\nu), \mathbf{u}(\nu)) \in H^{r}(\Omega)^{d \times d} \times H^{r}(\Omega) \times H^{r}(\Omega)^{d}$ for $1 \leq r \leq k+1$. Then there exists a constant $C>0$, independent of $h$, such that

$$
\left\|\boldsymbol{\sigma}(\nu)-\boldsymbol{\sigma}_{h}(\nu)\right\|_{0,3}+\left\|p(\nu)-p_{h}(\nu)\right\|_{0,3}+\left\|\mathbf{u}(\nu)-\mathbf{u}_{h}(\nu)\right\|_{0,3}
$$

$$
\leq C h^{r-\alpha}\left(\|\boldsymbol{\sigma}(\nu)\|_{r, 2}+\|p(\nu)\|_{r, 2}+\|\mathbf{u}(\nu)\|_{r, 2}\right)
$$

for any $\nu \in \Lambda$.

Proof. To show the validity of the first part of the theorem, we simply verify the assumptions in Theorem 3.3 of 24 or Theorem 4.1 of [16. First, it is easy to check that the operator $G$ satisfies the hypothesis (2) of Theorem 4.1 of [16]. The hypotheses (1) and (4) of Theorem 4.1 of [16] are proved in Theorems 5.5 and 5.2, respectively. By the definition of the space $\mathbf{Z}=\mathbf{Y}$, now it suffices to show

$$
D_{\phi} G(\nu, \phi) \in \mathcal{L}(\mathbf{X}, \mathbf{Z}) .
$$

To this end, for $(\mathbf{f}, \mathbf{g}) \in \mathbf{Y}$, by Theorem 4.5, the unique solution of (4.3), $\phi=$ $(\boldsymbol{\sigma}, p, \mathbf{u})$, is in $H^{1}(\Omega)^{d \times d} \times H^{1}(\Omega) \times H^{1}(\Omega)^{d}$ which is continuously imbedded in $L^{6}(\Omega)^{d \times d} \times L^{6}(\Omega) \times L^{6}(\Omega)^{d}$. By the triangle and Hölder inequalities, for any $\psi=$ $(\boldsymbol{\tau}, q, \mathbf{v}) \in \mathbf{X}$, we have

$$
\begin{aligned}
\| \mathbf{u} \cdot & (\boldsymbol{\tau}+q \boldsymbol{\delta})+\mathbf{v} \cdot(\boldsymbol{\sigma}+p \boldsymbol{\delta}) \|_{0,2} \\
& \leq\|\mathbf{u} \cdot(\boldsymbol{\tau}+q \boldsymbol{\delta})\|_{0,2}+\|\mathbf{v} \cdot(\boldsymbol{\sigma}+p \boldsymbol{\delta})\|_{0,2} \\
& \leq\|\mathbf{u}\|_{0,3}\left(\|\boldsymbol{\tau}\|_{0,3}+\|q\|_{0,3}\right)+\|\mathbf{v}\|_{0,3}\left(\|\boldsymbol{\sigma}\|_{0,6}+\|p\|_{0,6}\right) \\
& \leq\|\mathbf{u}\|_{1,2}\left(\|\boldsymbol{\tau}\|_{0,3}+\|q\|_{0,3}\right)+\|\mathbf{v}\|_{0,3}\left(\|\boldsymbol{\sigma}\|_{1,2}+\|p\|_{1,2}\right)
\end{aligned}
$$

This proves (5.18) and, hence, the well-posedness of the discrete problem in (5.13) by Theorem 4.1 of [16] or Theorem 3.3 of [24].

Assume $(\boldsymbol{\sigma}(\nu), p(\nu), \mathbf{u}(\nu)) \in H^{r}(\Omega)^{d \times d} \times H^{r}(\Omega) \times H^{r}(\Omega)^{d}$ with $1 \leq r \leq k+1$. The error estimate in (5.17) follows from Theorem 5.2. This completes the proof of the theorem.

\section{Cell-Centered finite volume scheme}

The mixed finite element method based on the pseudostress-velocity-pressure formulation usually leads to a larger system of algebraic equations than that resulting from the velocity-pressure formulation. To reduce its size, we eliminate the pseudostress unknowns through the hybridization technique (see [22, 1]). The reduced system is corresponding to a cell-centered finite volume scheme that preserves local balance of momentum. This hybridization technique was used in [12] to derive higher-order finite volume methods for second-order elliptic equations.

In this section, without going through derivation, we simply describe a finite volume scheme resulting from the rectangular RT element of the lowest order. To this end, for simplicity, assume that $\Omega$ is the unit square $(0,1) \times(0,1) \in \mathcal{R}^{2}$. Let $\mathcal{T}_{h}$ be the partition of $\Omega$ into sub-squares with the side length $h=N^{-1}$ :

$$
\bar{\Omega}=\bigcup_{i, j=1}^{N} \bar{K}_{i j} \quad \text { with } \quad \bar{K}_{i j}=\left[x_{i-1}, x_{i}\right] \times\left[y_{j-1}, y_{j}\right]=[(i-1) h, i h] \times[(j-1) h, j h] .
$$


Following the idea of [1, 12, we use interelement Lagrange multiplier to localize (hybridize) the mixed finite element problem in (3.6) with the lowest order element:

$$
X_{k}^{d} \times D_{k}^{d}=\left(\hat{R T_{0}^{2}} \times P_{0}\right) \times P_{0}^{2} .
$$

For any $\mathbf{w} \in R T_{0}$ and any $e \in \mathcal{E}_{h}$, note that $\left.\left(\mathbf{w} \cdot \mathbf{n}_{e}\right)\right|_{e} \in P_{0}(e)$. Let

$$
\Lambda_{0}=\left\{\lambda: \forall e \in \mathcal{E}_{h}, \lambda_{e} \in P_{0}(e)\right\} \quad \text { and } \quad \tilde{R T_{0}}=\left\{\mathbf{w} \in L^{2}(\Omega)^{2}:\left.\mathbf{w}\right|_{K} \in R T_{0}(K)\right\},
$$

then (3.6) can be localized by seeking a $\left(\boldsymbol{\sigma}_{h}, p_{h}, \mathbf{u}_{h}, \boldsymbol{\lambda}_{h}\right) \in \tilde{R T_{0}^{2}} \times P_{0} \times P_{0}^{2} \times \Lambda_{0}^{2}$ such that for all $K \in \mathcal{T}_{h}$,

$$
\left\{\begin{array}{l}
\left(\boldsymbol{\sigma}_{h}, \boldsymbol{\tau}\right)_{K}+\left(p_{h} \boldsymbol{\delta}, \boldsymbol{\tau}\right)_{K}+\left(\nabla \cdot \boldsymbol{\tau}, \mathbf{u}_{h}\right)_{K} \\
\quad-\nu\left(\boldsymbol{\tau} \cdot \mathbf{n}, \boldsymbol{\lambda}_{h}\right)_{\partial K \backslash \partial \Omega}=\nu<\boldsymbol{\tau} \cdot \mathbf{n}, \mathbf{g}>_{\partial K \cap \partial \Omega}, \\
\left(\operatorname{tr} \boldsymbol{\sigma}_{h}, q\right)_{K}+d\left(p_{h}, q\right)_{K}=0, \\
\left(\nabla \cdot \boldsymbol{\sigma}_{h}, \mathbf{v}\right)_{K}=-(\mathbf{f}, \mathbf{v})_{K}, \\
\sum_{K \in \mathcal{T}_{h}}<\boldsymbol{\sigma}_{h} \cdot \mathbf{n}, \boldsymbol{\mu}>_{\partial K \backslash \partial \Omega}=0, \\
\sum_{K \in \mathcal{T}_{h}}\left(p_{h}, 1\right)_{K}=0
\end{array}\right.
$$

for all $\boldsymbol{\tau} \in R T_{0}^{2}(K), q \in P_{0}(K), \mathbf{v} \in P_{0}(K)^{2}$, and $\boldsymbol{\mu} \in \Lambda_{0}^{2}$. The last equation in (6.1) followed from the second equation of (6.1) with $q=1$ and the condition $\int_{\Omega} \operatorname{tr} \sigma_{h} d x=0$.

The pseudostress variables $\boldsymbol{\sigma}_{h}$ in (6.1) can be eliminated by diagonalizing the mass matrix resulting in the inner product $(\boldsymbol{\sigma}, \boldsymbol{\tau})_{K}$ through an appropriate quadrature rule. The Lagrange multiplier introduced in the hybridization process may be eliminated by employing the consistency relations across the interfaces. The reduced system is then a cell-centered finite volume scheme for the Stokes equations in terms of the degrees of freedom on the velocity and pressure. For a detailed procedure of derivation, see [12] for elliptic second-order partial differential equations.

Here, we simply present our finite volume scheme obtained through this procedure. To this end, for an interior cell $K_{c}=K_{i j}=\left[x_{i-1}, x_{i}\right] \times\left[y_{j-1}, y_{j}\right]$ with $i, j \neq 1$ or $N$, denote its surrounding (left, right, top, and bottom) elements by $K_{l}$. $K_{r}$, $K_{t}$, and $K_{b}$, respectively (see Figure 1). Denote by $\tilde{\mathbf{u}}_{K}=\left(\tilde{u}_{1, K}, \tilde{u}_{2, K}\right)$ and $\tilde{p}_{K}$ the constant approximations of $\mathbf{u}$ and $p$ on the cell $K$. Then the cell-centered finite volume scheme is as follows:

$$
\left\{\begin{array}{c}
\nu\left(4 \tilde{u}_{1, K_{c}}-\tilde{u}_{1, K_{l}}-\tilde{u}_{1, K_{r}}-\tilde{u}_{1, K_{t}}-\tilde{u}_{1, K_{b}}\right) \\
+\frac{h}{2}\left(\tilde{p}_{K_{r}}-\tilde{p}_{K_{l}}\right)=\int_{K_{c}} f_{1} d x d y, \\
\nu\left(4 \tilde{u}_{2, K_{c}}-\tilde{u}_{2, K_{l}}-\tilde{u}_{2, K_{r}}-\tilde{u}_{2, K_{t}}-\tilde{u}_{2, K_{b}}\right) \\
+\frac{h}{2}\left(\tilde{p}_{K_{t}}-\tilde{p}_{K_{b}}\right)=\int_{K_{c}} f_{2} d x d y, \\
\frac{\nu}{2 h}\left(\left(\tilde{u}_{1, K_{r}}-\tilde{u}_{1, K_{l}}\right)+\left(\tilde{u}_{2, K_{t}}-\tilde{u}_{2, K_{b}}\right)\right) \\
+\frac{1}{4}\left(4 \tilde{p}_{K_{c}}-\tilde{p}_{K_{l}}-\tilde{p}_{K_{r}}-\tilde{p}_{K_{t}}-\tilde{p}_{K_{b}}\right)=0 .
\end{array}\right.
$$




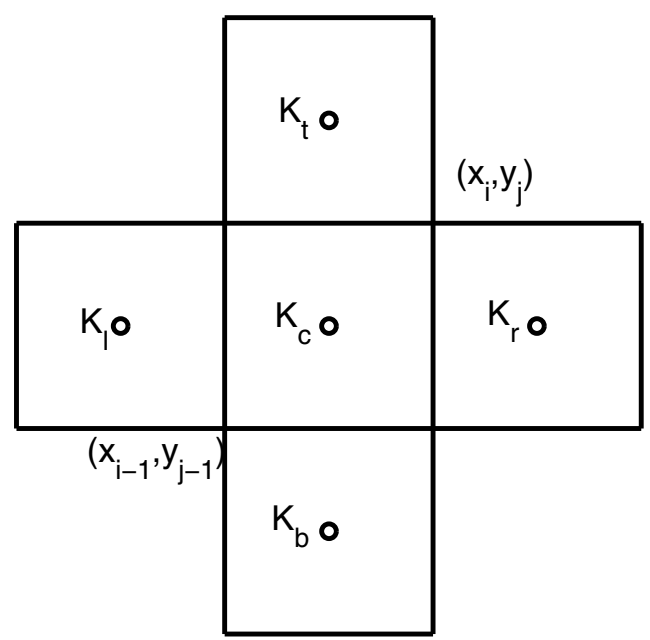

Figure 1. Elements

For boundary elements, similar formulae may be obtained. For example, let $K_{c}$ be an element whose right edge is on the boundary, and the remaining edges of the element are interior edges. Denote by $K_{l}, K_{t}$, and $K_{b}$ the adjacent elements sharing the left, top, and bottom edges, respectively, then the finite volume scheme for this cell is as follows:

$$
\begin{aligned}
& \int \nu\left(5 \tilde{u}_{1, K_{c}}-\tilde{u}_{1, K_{l}}-\tilde{u}_{1, K_{t}}-\tilde{u}_{1, K_{b}}\right)+\frac{h}{2}\left(\tilde{p}_{K_{c}}-\tilde{p}_{K_{l}}\right) \\
& =\int_{K_{c}} f_{1} d x d y+\frac{2 \nu}{h^{2}} \int_{\partial K_{c} \cap \partial \Omega} g_{1} d s, \\
& \nu\left(5 \tilde{u}_{2, K_{c}}-\tilde{u}_{2, K_{l}}-\tilde{u}_{2, K_{t}}-\tilde{u}_{2, K_{b}}\right)+\frac{h}{2}\left(\tilde{p}_{K_{t}}-\tilde{p}_{K_{b}}\right) \\
& =\int_{K_{c}} f_{2} d x d y+\frac{2 \nu}{h^{2}} \int_{\partial K_{c} \cap \partial \Omega} g_{2} d s, \\
& \frac{\nu}{2 h}\left(-\left(\tilde{u}_{1, K_{c}}+\tilde{u}_{1, K_{l}}\right)+\left(\tilde{u}_{2, K_{t}}-\tilde{u}_{2, K_{b}}\right)\right)+\frac{1}{4}\left(3 \tilde{p}_{K_{c}}-\tilde{p}_{K_{l}}-\tilde{p}_{K_{t}}-\tilde{p}_{K_{b}}\right) \\
& =-\frac{\nu}{h^{2}} \int_{\partial K_{c} \Gamma \partial \gamma_{2}} g_{1} d s \text {. }
\end{aligned}
$$

The finite volume scheme obtained above may be interpreted as a cell-centered finite volume scheme for the stabilized Stokes equation

$$
\begin{cases}-\nu \Delta \mathbf{u}+\nabla p=\mathbf{f} & \text { in } \Omega, \\ \nabla \cdot \mathbf{u}-h^{2} \Delta p= & \text { in } \Omega\end{cases}
$$


with boundary condition (1.2). More specifically, integrating the above equations over the cell $K_{c}=K_{i j}$ and using the Gauss divergence theorem gives

$$
\begin{cases}-\nu \int_{\partial K_{c}} \nabla u_{1} \cdot \mathbf{n} d s+\int_{y_{j-1}}^{y_{j}}\left(p\left(x_{i}, y\right)-p\left(x_{i-1}, y\right)\right) d y & =\int_{K_{c}} f_{1} d x d y \\ -\nu \int_{\partial K_{c}} \nabla u_{2} \cdot \mathbf{n} d s+\int_{x_{i-1}}^{x_{i}}\left(p\left(x, y_{j}\right)-p\left(x, y_{j-1}\right)\right) d x & =\int_{K_{c}} f_{2} d x d y \\ \int_{\partial K_{c}} \mathbf{u} \cdot \mathbf{n} d s-h^{2} \int_{\partial K_{c}} \nabla p \cdot \mathbf{n} d s & =0 .\end{cases}
$$

The finite volume scheme is then derived from a standard finite difference approximation of the above equations.

For the stationary Navier-Stokes equations, the derivation of the cell-centered finite volume scheme is identical. In particular, the stress is solved in terms of the velocity and the pressure. Hence, the nonlinear term, $-\frac{1}{\nu} \tilde{\mathbf{u}}_{h} \cdot\left(\tilde{\boldsymbol{\sigma}}_{h}+\tilde{p}_{h} \boldsymbol{\delta}\right)$, can be represented by the velocity and pressure. Let

$$
\mathbf{N}\left(\tilde{\mathbf{u}}_{h}, \tilde{p}_{h}\right)=\left(N_{1}, N_{2}\right)^{t} \equiv-\frac{1}{\nu} \tilde{\mathbf{u}}_{h} \cdot\left(\tilde{\boldsymbol{\sigma}}_{h}+\tilde{p}_{h} \boldsymbol{\delta}\right) .
$$

Then for an interior cell $K_{c}$, we have

$$
\left(\begin{array}{c}
N_{1} \\
N_{2}
\end{array}\right)=\left(\begin{array}{c}
\frac{h}{2}\left[\tilde{u}_{1, K_{c}}\left(\tilde{u}_{1, K_{r}}-\tilde{u}_{1, K_{l}}\right)+\tilde{u}_{2, K_{c}}\left(\tilde{u}_{1, K_{t}}-\tilde{u}_{1, K_{b}}\right)\right] \\
-\frac{h^{2}}{4 \nu} \tilde{u}_{1, K_{c}}\left(\tilde{p}_{K_{r}}+\tilde{p}_{K_{l}}-2 \tilde{p}_{K_{c}}\right) \\
\frac{h}{2}\left[\tilde{u}_{1, K_{c}}\left(\tilde{u}_{2, K_{r}}-\tilde{u}_{2, K_{l}}\right)+\tilde{u}_{2, K_{c}}\left(\tilde{u}_{2, K_{t}}-\tilde{u}_{2, K_{b}}\right)\right] \\
-\frac{h^{2}}{4 \nu} \tilde{u}_{2, K_{c}}\left(\tilde{p}_{K_{t}}+\tilde{p}_{K_{b}}-2 \tilde{p}_{K_{c}}\right)
\end{array}\right) .
$$

A cell-centered finite volume scheme for the stationary Navier-Stokes equations on an interior cell $K_{c}$ is as follows:

$$
\left\{\begin{aligned}
\nu\left(4 \tilde{u}_{1, K_{c}}-\tilde{u}_{1, K_{l}}-\tilde{u}_{1, K_{r}}-\tilde{u}_{1, K_{t}}-\tilde{u}_{1, K_{b}}\right)+N_{1} \\
+\frac{h}{2}\left(\tilde{p}_{K_{r}}-\tilde{p}_{K_{l}}\right)=\int_{K_{c}} f_{1} d x, \\
\nu\left(4 \tilde{u}_{2, K_{c}}-\tilde{u}_{2, K_{l}}-\tilde{u}_{2, K_{r}}-\tilde{u}_{2, K_{t}}-\tilde{u}_{2, K_{b}}\right)+N_{2} \\
+\frac{h}{2}\left(\tilde{p}_{K_{t}}-\tilde{p}_{K_{b}}\right)=\int_{K_{c}} f_{2} d x, \\
\frac{\nu}{2 h}\left(\left(\tilde{u}_{1, K_{r}}-\tilde{u}_{1, K_{l}}\right)+\left(\tilde{u}_{2, K_{t}}-\tilde{u}_{2, K_{b}}\right)\right) \\
+\frac{1}{4}\left(4 \tilde{p}_{K_{c}}-\tilde{p}_{K_{l}}-\tilde{p}_{K_{r}}-\tilde{p}_{K_{t}}-\tilde{p}_{K_{b}}\right)=0 .
\end{aligned}\right.
$$

Schemes for boundary elements can be obtained in a similar fashion.

Remark 6.1. The finite volume schemes derived in this section for the Stokes and Navier-Stokes equations are identical to the corresponding mixed finite element methods with numerical integration. Since the numerical integration does not decrease the order of convergence of the original mixed methods, the finite volume schemes have the same approximation accuracy as the corresponding mixed methods. 


\section{REFERENCES}

[1] D.N. ARnold AND F. BREzZI, Mixed and nonconforming finite element methods: implementation, postprocessing and error estimates, M2AN, 19 (1985), 7-32. MR813687 (87g:65126)

[2] D. N. Arnold, J. Douglas, JR., And C. P. Gupta, A family of higher order mixed finite element methods for plane elasticity, Numer. Math., 45 (1984), 1-22. MR761879(86a:65112)

[3] D. N. Arnold, R. S. Falk, And R. Winther, Preconditioning in H(div) and applications, Math. Comp., 66:219 (1997), 957-984. MR1401938 (97i:65177)

[4] D. N. Arnold, R. S. Falk, And R. Winther, Multigrid in $H$ (div) and $H$ (curl), Numer. Math., 85 (2000), 197-218. MR.1754719(2001d:65161)

[5] M.A. Behr, L.P. Franca, And T.E. Tezduyar, Stabilized finite element methods for the velocity-pressure-stress formulation of incompressible flows, Comput. Methods Appl. Mech. Eng., 104 (1993), 31-48. MR.1210650 (94d:76051)

[6] F. BREzzI, On existence, uniqueness and approximation of saddle-point problems arising from Lagrange multipliers, RAIRO Anal. Numér., 2(1974), 129-151. MR0365287 (51:1540)

[7] F. Brezzi, J. Douglas, JR., R. Duran, and M. Fortin, Mixed finite elements for second order elliptic problems in three variables, Numer. Math., 51(1987), 237-250. MR890035 (88f:65190)

[8] F. Brezzi, J. Douglas, JR., M. Fortin, and D. Marini, Efficient rectangular mixed finite elements in two and three space variables, $\mathrm{M}^{2} \mathrm{AN}, 21(1987), 581-604$. MR.921828 (88j:65249)

[9] F. Brezzi, J. Douglas, JR., AND D. MARIni, Two families of mixed finite elements for second order elliptic problems, Numer. Math., 47(1985), 217-235. MR799685 (87g:65133)

[10] F. Brezzi And M. Fortin, Mixed and Hybrid Finite Element Methods, Springer-Verlag, New York, 1991. MR.1115205 (92d:65187)

[11] F. BRezzi, J. RAPPAZ, AND P.A. Raviart, Finite-dimensional approximation of nonlinear problems, Part I: Branches of nonsingular solutions, Numer. Math., 36(1980), pp.1-25. MR.595803 (83f:65089a)

[12] Z. CAI, J. Douglas, JR., And M. PARK, Development and analysis of higher order finite volume methods over rectangles for elliptic equations, Advan. Comp. Math., 19:1-3 (2003), 3-33. MR 1973457 (2004g:65141)

[13] Z. CAI, B. LEE, AND P. WANG, Least-squares methods for incompressible Newtonian fluid flow: linear stationary problems, SIAM J. Numer. Anal., 42:2 (2004), 843-859. MR2084238 (2005i:65180)

[14] Z. CAI AND G. STARKe, First-order system least squares for the stress-displacement formulation: linear elasticity, SIAM J. Numer. Anal., 41:2 (2003), 715-730. MR2004196 (2005e:65180)

[15] Z. Cai, C. Tong, P. Vassilevski and C. Wang, Mixed finite element methods for incompressible flow: Stationary Stokes equations, Numer. Methods PDEs, 26:4 (2010), 957-978. MR2642330 (2011e:65259)

[16] Z. CAI, C. WAng, AND S. Zhang, Mixed finite element methods for incompressible flow: stationary Navier-Stokes equations, SIAM J. Numer. Anal., 48:1 (2010), 79-94. MR2608359 (2011i:65206)

[17] Z. CAi AND Y. WAng, A multigrid method for the pseudostress formulation of Stokes problems, SIAM J. Sci. Comput., 29 (2007), 2078-2095. MR2350022 (2008g:65156)

[18] P. Constantin and C. Foias, Navier-Stokes Equations, The University of Chicago Press, Chicago and London, 1988. MR972259 (90b:35190)

[19] M. Farhloul and M. Fortin, A new mixed finite element for the Stokes and elasticity problems, SIAM J. Numer. Anal., 30 (1993), 971-990. MR.1231323 (94g:65123)

[20] M. FARHLOUl And M. Fortin, Review and complements on mixed-hybrid finite element methods for fluid flows, J. Comp. Appl. Math., 140 (2002), 301-313. MR1934446 (2003k:65148)

[21] L. E. Figueroa, G. N. Gatica, And A. Marquez Augmented mixed finite element methods for the stationary Stokes equations, SIAM J. Sci. Comput. 31, (2008) 1082-1119. MR2466149 (2009i:65215)

[22] B. X. Fraeiss de Veubeke, Stress function approach, International Congress on the Finite Element Method in Structural Mechanics, Bournemouth, 1975.

[23] G. Gatica, M. González and S. Meddahi, A low-order mixed finite element method for a class of quasi-Newtonian Stokes flows, Comput. Methods Appl. Mech. Eng., 193 (2004), Part I: a priori error analysis, 881-892; Part II: a posteriori error analysis, 893-911. 
[24] V. Girault and P. A. Raviart, Finite Element Methods for Navier-Stokes Equations: Theory and Algorithms, Springer-Verlag, New York, 1986. MR851383 (88b:65129)

[25] M. D. Gunzburger, Finite Element Methods for Viscous Incompressible Flows, Academic Press, 1989. MR1017032 (91d:76053)

[26] R. Hiptmain, Multigrid method for H(div) in three dimensions, ETNA, 6 (1997), 133-152. MR.1615161 (99c:65232)

[27] F. A. Milner ANd E.J. PARK, Mixed finite-element methods for Hamilton-Jacobi-Bellmantype equations, IMA J. Numer. Anal., 16(3) (1996), 399-412. MR1397603 (97h:65143)

[28] J. C NÉDÉLEC, Mixed finite elements in $R^{3}$, Numer. Math., 35 (1980), 315-341. MR592160 (81k:65125)

[29] P. A. Raviart and I. M. Thomas, A mixed finite element method for second order elliptic problems, Lect. Notes Math. 606, Springer-Verlag, Berlin and New York (1977), 292-315. MR0483555 (58:3547)

[30] P. S. VASSILEVSKI AND J. WANG, Multilevel iterative methods for mixed finite element discretizations of elliptic problems, Numer. Math., 63 (1992), 503-520. MR.1189534 (93j:65187)

Department of Mathematics, Purdue University, 150 N. University Street West LAFAYETTE, INDIANA 47907-2067

E-mail address: zcai@math.purdue.edu

Division of Applied Mathematics, Brown University, Providence, Rhode Island 02912

E-mail address: Shun_Zhang@brown.edu 